\title{
AN INTERVAL FINITE ELEMENT METHOD FOR THE ANALYSIS OF STRUCTURES WITH SPATIALLY VARYING UNCERTAINTIES
}

\author{
Alba Sofi ${ }^{\mathrm{a}, \mathrm{b}^{*}}$, Eugenia Romeo ${ }^{\mathrm{c}}$, Olga Barrera ${ }^{\mathrm{d}, \mathrm{e}}$, Alan Cocks $^{\mathrm{e}}$ \\ ${ }^{a}$ Department of Architecture and Territory and Inter-University Centre of Theoretical and Experimental \\ Dynamics, University "Mediterranea" of Reggio Calabria Salita Melissari, Feo di Vito, 89124 Reggio Calabria \\ Italy. E-mail: alba.sofi@unirc.it \\ ${ }^{b}$ Visiting Fellow at the Department of Engineering Science, University of Oxford, Parks Road, OX13PJ, Oxford \\ (UK) \\ 'Department of Civil, Energy, Environmental and Materials Engineering, University "Mediterranea" of Reggio \\ Calabria, Via Graziella, Località Feo di Vito, 89124 Reggio Calabria, Italy. E-mail: eugenia.romeo@unirc.it \\ ${ }^{\mathrm{d} S}$ School of Engineering, Computing and Mathematics, Oxford Brookes University, Wheatley Campus, Wheatley, \\ Oxon (UK) OX33 1HX. E-mail: obarrera@brookes.ac.uk \\ ${ }^{\mathrm{e}}$ Department of Engineering Science, University of Oxford, Parks Road, OX13PJ, Oxford (UK). E-mail: \\ alan.cocks@eng.ox.ac.uk
}

\section{Abstract}

Finite element analysis of linear-elastic structures with spatially varying uncertain properties is addressed within the framework of the interval model of uncertainty. Resorting to a recently proposed interval field model, the uncertain properties are expressed as the superposition of deterministic basis functions weighted by particular unitary intervals. An Interval Finite Element Method (IFEM) incorporating the interval field representation of uncertainties is formulated by applying an interval extension in conjunction with the standard energy approach. Uncertainty propagation analysis is performed by adopting a response surface approach which provides approximate explicit expressions of response bounds requiring only a few deterministic analyses. Then, the whole procedure is implemented in ABAQUS' environment by coding User Subroutines and Python scripts.

2D plane stress and bending problems involving uncertain Young's modulus of the material are analyzed. The accuracy of the proposed IFEM as well as response variability under spatially dependent uncertainty are investigated.

Keywords: finite element method, interval field, response surface approach, lower bound and upper bound, ABAQUS

*Corresponding author 


\section{INTRODUCTION}

Uncertainty assessment of structural systems is attracting growing interest in various engineering and industrial fields. Indeed, it is widely recognized that uncertainties affecting model parameters, such as material or geometrical properties, may have a significant influence on the performance of engineering systems [1],[2]. One of the most urgent challenges in the context of non-deterministic analysis is to develop efficient and robust algorithms for predicting the influence of uncertain parameters on the response of real world large-scale systems. Over the last decades, much research effort has been devoted to incorporating uncertainties into the standard finite element method (FEM) in order to exploit all capabilities of deterministic FE solvers as well as the increasing power of available computational resources.

The extension of the classical FEM to problems involving uncertainties modeled as random variables or random fields led to the well-known Stochastic Finite Element Method (SFEM), which may be viewed nowadays as the most powerful tool in the field of computational stochastic mechanics (see e.g., [3],[4]). Many variants of the SFEM have been proposed in the literature, while much less attention has been devoted to the development of specialized software for the analysis of large-scale stochastic problems. To address the need for probabilistic FE analysis in practical engineering, ANSYS Inc. released two tools, namely the ANSYS Probabilistic Design System and the ANSYS DesignXplorer [5]. Such tools enable random input variables to be accounted for, such as material properties, boundary conditions, loads and geometry, and to handle several types of analysis. However, the description of uncertainties is limited to the use of random variables. A random field [6] representation is required to take into account the inherent spatial dependency of non-deterministic properties which may significantly affect the reliability of a design. A stochastic FE library (StoFEL) has also been coupled with ANSYS for predicting response variability [7]. Among specialized software for SFE anal- 
ysis, it is worth mentioning computational stochastic structural analysis (COSSAN) [8], numerical evaluation of stochastic structures under stress (NESSUS) [9], and finite element reliability using MATLAB (FERUM) [10]. To the extent of the author's knowledge, only Shang et al. [11] faced the challenging task of incorporating uncertain mechanical properties modeled as homogeneous random fields into commercial finite element programmes such as ABAQUS by coding a User Element subroutine (UEL). Though well-established, the concept of a random field has not yet been implemented in commercial FE software.

Since the mid-1990s, non-probabilistic models of uncertainty have been used in the context of FE analysis which are complementary rather than competitive to the traditional probabilistic description, leading to the formulation of the so-called Interval FEM (IFEM) and Fuzzy FEM (FFEM) [12],[13] approaches. The IFEM and FFEM describe the uncertain input parameters as interval variables [14]-[16] and fuzzy sets [17], respectively. Interval variables are characterized by assigned lower bounds and upper bounds, while the fuzzy set concept also provides information about the level of membership of a certain value to the range of possible input values. The main feature of these models is that they do not require a complete probabilistic characterization of uncertainties which implies the availability of a large amount of data. Furthermore, propagation of uncertainty through numerical algorithms is usually less time consuming. Currently, research activities mainly focus on IFEMs since, based on the $\alpha$-level technique, the fuzzy analysis reduces to the consecutive solution of a number of interval problems [13]. The IFEM may be viewed as a useful computational tool in early design stages when available information is generally insufficient to perform a probabilistic analysis [12]. While the SFEM is well-established and accepted by the scientific community, much research efforts are still needed to further enhance the development and dissemination of the IFEM in practical engineering. Several versions of the IFEM have been proposed in the literature (see e.g., [18][24]) with the purpose of addressing the following three key issues: $i$ ) the overestimation of the 
interval solution range due to the so-called dependency phenomenon, which typically affects methods based on the Classical Interval Analysis (CIA) [16]; ii) the inherent spatial character of uncertainties, like material or geometric properties, which is not taken into account by traditional IFEMs ([18]-[24]); iii) the need for computationally efficient propagation procedures.

Among the approaches proposed in the literature to limit the effects of the dependency phenomenon, the so-called improved interval analysis via extra unitary interval (IIA via EUI) [25] has proved to be an effective remedy to reduce conservatism in the context of interval structural analysis. This approach relies on the use of a particular unitary interval, called EUI, which does not follow the rules of the CIA.

To describe the spatial character of interval uncertainties, the interval field model [26],[27] has been recently introduced as the natural extension of the random field concept to the nonprobabilistic framework. The interval field description of an uncertain property basically consists of a superposition of deterministic basis functions representing the spatial character, weighted by independent interval coefficients representing the uncertainty. Different definitions of the interval field have been introduced in the literature, such as those based on the Inverse Distance Weighting interpolation (IDW) or the Local Interval Field Decomposition method (LIFD) [28]. Recently, an interval field model based on the IIA via EUI [29] has been proposed with the purpose of handling both overestimation and spatial dependency issues. This model expresses the generic uncertain property as superposition of deterministic functions and EUIs. To date, applications of the interval field model based on the IIA via EUI are limited to the static analysis of one-dimensional problems involving spatially varying uncertain Young's modulus [29]-[31]. Faes and Moens [32] presented a novel methodology for the identification and quantification of spatial uncertainty modelled as an interval field, based on a large set of measurement data, by extending a recently proposed method to identify interval scalars [33]. 
So far, very few studies have been devoted to the incorporation of the interval field model into the standard FEM (see e.g., [27], [34]-[36]). Furthermore, the development of specialized software, able to interact with powerful third-party software for the analysis of large-scale problems with uncertain properties modeled either as interval variables or interval fields, has not been addressed in the scientific literature.

To fill this gap, the present paper deals with the formulation and implementation of an IFEM for the analysis of structures made of linear-elastic isotropic materials with spatially varying uncertainties described using the interval field model based on the IIA via EUI. Without loss of generality, only Young's modulus of the material is assumed to be uncertain. The key idea is to incorporate the interval field representation of the uncertain material property into the standard FEM by defining the pertinent interval element constitutive matrix which depends on the spatial coordinates as well as on a certain number of EUIs. Then, interval extension of the standard energy approach and the conventional assembly procedure yield the set of linear interval equations governing the interval global displacements of the FE model. Within the interval framework, the solution of such equations is pursued by evaluating the bounds of the interval displacement vector. To achieve this aim, an efficient procedure based on the application of a ratio of polynomial response surface in conjunction with the IIA via EUI is adopted. The challenging task of evaluating the bounds of the interval stress components is also addressed by exploiting the response surface approximation combined either with a sensitivity or a combinatorial approach. The proposed response surface strategy for propagating the interval field requires only a certain number of deterministic analyses, thus allowing a significant reduction of the computational burden compared to the classical combinatorial procedure, known as the vertex method [37].

The main feature of the described IFEM, incorporating the interval field model of Young's modulus, lies in its capability of interacting non-intrusively with a commercial FE code thus 
providing a powerful tool for the analysis of real engineering problems. Indeed, the basic steps of the IFEM formulation are the same as those of the standard FEM, and the proposed uncertainty propagation strategy requires repeated deterministic analyses which can be efficiently performed by a FE solver. In view of these observations, one of the main purposes of the present study is to integrate the proposed IFEM into the commercial FE software ABAQUS. This challenging task is pursued by implementing User MATerial (UMAT) or USerDefinedFieLD (USDFLD) subroutines [38] in the FORTRAN 77 language and Python scripts which enable the interval Young's modulus field to be incorporated into the constitutive behaviour of any type of FE available in ABAQUS' library. To the best of the authors' knowledge, this is the first attempt to integrate the interval field model of uncertainty into commercial FE software. The approach adopted here can be readily employed to develop similar routines for other commercial FE packages.

Numerical results concerning 2D plane stress and bending problems with uncertain Young's modulus are presented. The accuracy and efficiency of the proposed IFEM are demonstrated by suitable comparisons with the bounds of the response provided by the vertex method.

The paper is organized as follows: Section 2 outlines the formulation of the IFEM incorporating the uncertain Young's modulus described as an interval field based on the IIA via EUI; Section 3 is devoted to the development of response surface based strategies for evaluating the bounds of the interval displacements and stresses; Section 4 focuses on the implementation of the IFEM into the commercial FE software ABAQUS; finally, in Section 5, two numerical applications are presented.

\section{INTERVAL FINITE ELEMENT METHOD INCORPORATING SPATIALLY}

\section{VARYING UNCERTAINTIES}


The underlying idea of the interval model, originally developed from the interval analysis $[14],[16]$, is to describe the generic uncertain parameter as an interval variable with given lower bound (LB) and upper bound (UB). This model is very useful when only the range of variability of the uncertain parameters is known, while available information is insufficient to define the type of distribution within the range, as often happens in the early stages of design.

The so-called Interval Finite Element Method (IFEM) has been developed as an extension of the traditional FEM by incorporating uncertain input parameters modeled as interval variables (see e.g., [12],[13]). The standard formulation of the IFEM relies on the extreme assumption of total spatial independency (TSI) of uncertainties. Specifically, a spatially varying uncertain property, such as Young's modulus of the material, is represented as a set of interval variables, one for each FE. This assumption may lead to serious shortcomings such as overestimation of the actual uncertainty, mesh-dependency of the solution and increase of the computational effort [23]. Furthermore, interval variables are by definition unable to account for mutual dependency between the values of spatially varying properties at different locations. Alternatively, relying on the opposite extreme hypothesis of total spatial dependency (TSD), the uncertain property can be described as a single interval variable over the entire model.

A more realistic and computationally efficient description of spatially varying interval uncertainties can be obtained by resorting to the interval field model [26],[27], which is able to quantify the dependency between adjacent values of an interval quantity that cannot differ as much as values that are further apart. The key idea is to represent the spatial character and the uncertainty separately by expressing an uncertain property as a superposition of deterministic basis functions weighted by independent interval coefficients. In the context of a FE formulation, the interval field model enables the spatial dependency of the uncertainty properties to be taken into account as well as to drastically reduce the dimensionality of the uncertainty. Indeed, 
the latter is not related to the number of FEs of the selected mesh, as in the standard IFEM, but it is given by the number of series terms retained in the interval field representation.

High dimensionality of uncertainty may have negative effects on both the computational efficiency and accuracy of IFE procedures. Indeed, one of the main drawbacks of IFEMs based on the Classical Interval Analysis (CIA) [14] is the overestimation of the interval solution range due to the so-called dependency phenomenon [16], which increases tremendously with the number of interval variables involved and the number of interval computations. Hence, within the interval framework, it is highly desirable to reduce the dimensionality of uncertainty.

To overcome the main limitations arising from the use of discrete interval variables in the context of FE analysis, in the present study, a novel IFEM incorporating the interval field description of spatially varying uncertainties is developed.

\subsection{Uncertain Young's modulus modeled as an interval field}

Let us consider a continuous body made of a linear-elastic isotropic material which occupies the volume $V$ bounded by the surface $S$ in its undeformed state. The body is subjected to volume forces $\mathbf{b}(\mathbf{x})$ in $V$ and surface forces $\mathbf{t}(\mathbf{x})$ on the free portion $S_{t}$ of the boundary surface $S$, with $\mathbf{x}$ denoting the position vector of a generic point referred to a Cartesian coordinate system $O(x, y, z)$; the displacements $\mathbf{u}_{u}(\mathbf{x})$ are imposed on the constrained portion $S_{u}$ of $S$. The loads act by hypothesis in a quasi-static manner and infinitesimal displacements are considered. Without loss of generality, all input parameters are assumed to be deterministic, except Young's modulus of the material which is treated as an uncertain property in the context of the interval model of uncertainty. In order to take into account the inherent spatial dependency of continuous material properties, the uncertain Young's modulus is described resorting to a recently proposed interval field model [29] based on the Improved Interval Analysis (IIA via EUI) 
[25]. The main features of the assumed interval field model are herein briefly summarized. Let Young's modulus be represented by the following interval function:

$$
E^{I}(\mathbf{x})=[\underline{E}(\mathbf{x}), \bar{E}(\mathbf{x})]=E_{0}\left[1+B^{I}(\mathbf{x})\right], \quad \mathbf{x} \in V
$$

with midpoint and deviation amplitude given, respectively, by:

$$
\operatorname{mid}\left\{E^{I}(\mathbf{x})\right\}=\frac{\bar{E}(\mathbf{x})+\underline{E}(\mathbf{x})}{2} \equiv E_{0} ; \quad \Delta E(\mathbf{x})=\frac{\bar{E}(\mathbf{x})-\underline{E}(\mathbf{x})}{2} \equiv E_{0} \Delta B(\mathbf{x}), \quad \mathbf{x} \in V .
$$

In the previous equations, the superscript $I$ denotes interval quantities; $\underline{E}(\mathbf{x})$ and $\bar{E}(\mathbf{x})$ are the lower bound (LB) and upper bound (UB) functions; the operator mid $\{\bullet\}$ yields the midpoint of the interval quantity between curly brackets; $B^{I}(\mathbf{x})=[\underline{B}(\mathbf{x}), \bar{B}(\mathbf{x})]$ is a dimensionless interval function with zero midpoint and deviation amplitude $\Delta B(\mathbf{x})<1$, so as to ensure values of the uncertain material property are always positive. Notice that the midpoint value of $E^{I}(\mathbf{x})$, herein assumed constant over the volume $V$, coincides with the nominal value of the uncertain Young's modulus $E_{0} \in \mathbb{R}$ (see Eq. (2a)).

The key idea behind the interval field model based on the IIA via EUI is to describe the spatial dependency of the uncertain property by introducing the following real, deterministic, symmetric, non-negative function:

$$
\Gamma_{B}(\mathbf{x}, \xi)=\operatorname{mid}\left\{B^{I}(\mathbf{x}) B^{I}(\xi)\right\} \equiv \frac{\operatorname{mid}\left\{E^{I}(\mathbf{x}) E^{I}(\xi)\right\}}{\left(E_{0}\right)^{2}}-1, \quad \mathbf{x}, \xi \in V
$$

called spatial dependency function. This function is intended to provide information on how similar the values of $B^{I}(\mathbf{x})$ at nearby locations of the domain are. If the $\operatorname{mid}\{\bullet\}$ operator is regarded as the analogue of the stochastic average operator [29], the function $\Gamma_{B}(\mathbf{x}, \xi)$ may be viewed as the non-probabilistic counterpart to the autocorrelation function characterizing 
random fields [6]. Specifically, the spatial dependency function provides a measure of the dependency between the values of the dimensionless interval function $B^{I}$ at different locations $\mathbf{x}$ and $\xi$. It may also be viewed as the relative difference between the midpoint of the product of Young's modulus at different locations, $\mathbf{x}$ and $\xi$, and the squared midpoint of the interval field $E_{0}$ (see Eq. (3)) assumed constant over the whole domain. The analytical expression of the spatial dependency function has to be postulated in a physically consistent way. For instance, an exponential or squared exponential form can be assumed. So far no measurementbased functions have been defined. Based on Eq. (3), experimental tests should be designed in such a way that the value of Young's modulus at different pairs of locations can be measured.

In the context of finite element (FE) formulations, it is useful to represent the continuous interval function $E^{I}(\mathbf{x})$ in terms of a set of independent interval coefficients. To this aim, the following spectral decomposition of the spatial dependency function is adopted:

$$
\Gamma_{B}(\mathbf{x}, \boldsymbol{\xi})=\sum_{i=1}^{\infty} \lambda_{i} \psi_{i}(\mathbf{x}) \psi_{i}(\boldsymbol{\xi}) \Rightarrow \Gamma_{B}(\mathbf{x}, \mathbf{x}) \equiv \operatorname{mid}\left\{\left(B^{I}(\mathbf{x})\right)^{2}\right\}=\sum_{i=1}^{\infty} \lambda_{i} \psi_{i}^{2}(\mathbf{x})
$$

where $\lambda_{i}$ and $\psi_{i}(\mathbf{x}),(i=1,2, \ldots)$, are the eigenvalues and associated eigenfunctions of $\Gamma_{B}(\mathbf{x}, \xi)$, which are solutions of the following homogeneous Fredholm integral equation of the second kind:

$$
\int_{V} \Gamma_{B}(\mathbf{x}, \xi) \psi_{i}(\mathbf{x}) \mathrm{d} \mathbf{x}=\lambda_{i} \psi_{i}(\xi)
$$

By introducing the so-called EUI [25], $\hat{e}_{i}^{I}=[-1,+1]$, and truncating the decomposition (4) to the first $M$ terms, the following Karhunen-Loève (KL)-like expansion of the dimensionless interval function $B^{I}(\mathbf{x})$ (see Eq. (1)) is obtained: 


$$
B^{I}(\mathbf{x})=\sum_{i=1}^{M} \sqrt{\lambda_{i}} \psi_{i}(\mathbf{x}) \hat{e}_{i}^{I}, \quad \mathbf{x} \in V
$$

Then, substituting Eq. (6) into Eq. (1), the interval field representation of the uncertain Young's modulus $E^{I}(\mathbf{x})$ based on the IIA via EUI is obtained [29]:

$$
E^{I}(\mathbf{x})=E_{0}\left[1+\sum_{i=1}^{M} \sqrt{\lambda_{i}} \psi_{i}(\mathbf{x}) \hat{e}_{i}^{I}\right], \quad \mathbf{x} \in V
$$

where the deterministic functions $\sqrt{\lambda_{i}} \psi_{i}(\mathbf{x})$ describe the spatial character, while the associated EUIs, $\hat{e}_{i}^{I}$, represent the uncertainty.

The LB and UB of the interval Young's modulus (7) are spatially dependent functions given by the following relationships:

$$
\underline{E}(\mathbf{x})=E_{0}[1-\Delta B(\mathbf{x})] ; \quad \bar{E}(\mathbf{x})=E_{0}[1+\Delta B(\mathbf{x})], \quad \mathbf{x} \in V
$$

where

$$
\Delta B(\mathbf{x})=\frac{\Delta E(\mathbf{x})}{E_{0}}=\sum_{i=1}^{M}\left|\sqrt{\lambda_{i}} \psi_{i}(\mathbf{x})\right|, \quad \mathbf{x} \in V
$$

with $|\bullet|$ denoting the absolute value of $\bullet$

It is worth remarking that the interval field model based on the IIA via EUI in Eq. (7) describes the spatial dependency and the uncertainty of Young's modulus separately by means of the deterministic functions $\sqrt{\lambda_{i}} \psi_{i}(\mathbf{x})$ and the associated EUIs, $\hat{e}_{i}^{I}$, respectively. This is a highly desirable feature in the framework of interval field representation (see e.g., [33]) which enables propagation techniques commonly used for discrete input interval variables to be applied. 
Taking into account Eq. (7), the interval constitutive matrix $\mathbf{D}^{I}(\mathbf{x})$ for the continuous body with interval Young's modulus can be expressed as follows:

$$
\mathbf{D}^{I}(\mathbf{x})=\mathbf{D}_{0}\left[1+\sum_{i=1}^{M} \sqrt{\lambda_{i}} \psi_{i}(\mathbf{x}) \hat{e}_{i}^{I}\right]=\mathbf{D}_{0}+\sum_{i=1}^{M} \mathbf{D}_{i}(\mathbf{x}) \hat{e}_{i}^{I}
$$

where $\mathbf{D}_{0}$ is the nominal constitutive matrix and $\mathbf{D}_{i}(\mathbf{x})=\sqrt{\lambda_{i}} \psi_{i}(\mathbf{x}) \mathbf{D}_{0}$ is the deviation matrix associated with the $i$-th EUI.

It is worth mentioning that the random field model remains the most valuable representation of spatially varying uncertainties when objective information on non-determinism is available and a probabilistic description of the output is desired. As known, the characterization of a random field requires a large amount of experimental data to define the probability density function and the correlation structure. Often, the lack of sufficient information leads to strong assumptions on the probabilistic characterization of the random field. Even small deviations from the true probabilistic model may have a high impact on reliability estimates. In these cases, the interval field model represents a suitable alternative since it does not require a complete probabilistic description of the uncertain property.

\subsection{Interval finite element formulation}

Let the body be subdivided into $N$ FEs of volume $V_{h}(h=1,2, \ldots, N)$. According to the standard displacement-based FE formulation, the interval displacement field within the $h-$ th FE can be approximated as follows:

$$
\mathbf{u}^{(h) I}(\mathbf{x})=\mathbf{N}^{(h)}(\mathbf{x}) \mathbf{d}^{(h) I}, \quad(h=1,2, \ldots, N)
$$


where $\mathbf{N}^{(h)}(\mathbf{x})$ is the matrix collecting the deterministic shape functions; $\mathbf{d}^{(h) I}$ is the interval vector listing the element nodal displacements.

Replacing Eq.(11) into the strain-displacement and linear-elastic constitutive equations yield, respectively, the following expressions of the interval strain and stress fields within the $h$-th FE:

$$
\begin{aligned}
\boldsymbol{\varepsilon}^{(h) I}(\mathbf{x}) & =\mathbf{B}^{(h)}(\mathbf{x}) \mathbf{d}^{(h) I} \\
\boldsymbol{\sigma}^{(h) I}(\mathbf{x}) & =\mathbf{D}^{I}(\mathbf{x}) \mathbf{B}^{(h)}(\mathbf{x}) \mathbf{d}^{(h) I}
\end{aligned}
$$

where $\mathbf{B}^{(h)}(\mathbf{x})$ is the strain-displacement matrix and $\mathbf{D}^{I}(\mathbf{x})$ denotes the interval constitutive matrix defined in Eq. (10). Notice that the interval stress field is affected by uncertainties both through the interval nodal displacements and the interval constitutive matrix. Multiple occurrences of the same interval variable in Eq. (12b) makes the stress field more sensitive to the dependency phenomenon than the displacement field [23].

By virtue of the interval extension [16] and taking into account Eqs. (11) and (12), the following discretized form of the Interval Total Potential Energy (ITPE) functional of the body is obtained:

$$
\begin{aligned}
\Pi\left[\mathbf{d}^{(h) I}\right]= & \Phi^{I}-L_{e}^{I}=\frac{1}{2} \sum_{h=1}^{N}\left(\mathbf{d}^{(h) I}\right)^{\mathrm{T}} \int_{V^{(h)}} \mathbf{B}^{(h) \mathrm{T}}(\mathbf{x}) \mathbf{D}^{I}(\mathbf{x}) \mathbf{B}^{(h)}(\mathbf{x}) \mathrm{d} V^{(h)} \mathbf{d}^{(h) I} \\
& -\sum_{h=1}^{N}\left(\mathbf{d}^{(h) I}\right)^{\mathrm{T}}\left(\int_{V^{(h)}} \mathbf{N}^{(h) \mathrm{T}}(\mathbf{x}) \mathbf{b}(\mathbf{x}) \mathrm{d} V^{(h)}+\int_{S_{f}^{(h)}} \mathbf{N}^{(h) \mathrm{T}}(\mathbf{x}) \mathbf{t}(\mathbf{x}) \mathrm{d} S^{(h)}\right)
\end{aligned}
$$

where $\Phi^{I}$ is the interval elastic strain energy stored in the deformed body, while $L_{e}^{I}$ is the interval work done by the external loads, expressed as sum of the contributions associated to each FE. Equation (13) can be rewritten as follows:

where

$$
\Pi\left[\mathbf{d}^{(h) I}\right]=\frac{1}{2} \sum_{h=1}^{N}\left(\mathbf{d}^{(h) I}\right)^{\mathrm{T}} \mathbf{k}^{(h) I} \mathbf{d}^{(h) I}-\sum_{h=1}^{N}\left(\mathbf{d}^{(h) I}\right)^{\mathrm{T}} \mathbf{f}^{(h)}
$$

$$
\mathbf{k}^{(h) I}=\int_{V^{(h)}} \mathbf{B}^{(h) \mathrm{T}}(\mathbf{x}) \mathbf{D}^{I}(\mathbf{x}) \mathbf{B}^{(h)}(\mathbf{x}) \mathrm{d} V^{(h)}
$$


is the interval element stiffness matrix, formally analogous to the one pertaining to the deterministic FE, and

$$
\mathbf{f}^{(h)}=\int_{V^{(h)}} \mathbf{N}^{(h) \mathrm{T}}(\mathbf{x}) \mathbf{b}(\mathbf{x}) \mathrm{d} V^{(h)}+\int_{S_{t}^{(h)}} \mathbf{N}^{(h) \mathrm{T}}(\mathbf{x}) \mathbf{t}(\mathbf{x}) \mathrm{d} S^{(h)}
$$

denotes the element force vector, which is not affected by uncertainty.

By substituting expression (10) for the constitutive matrix $\mathbf{D}^{I}(\mathbf{x})$ in Eq. (15), the interval element stiffness matrix can be recast as:

$$
\mathbf{k}^{(h) I}=\mathbf{k}_{0}^{(h)}+\sum_{i=1}^{M} \mathbf{k}_{i}^{(h)} \hat{e}_{i}^{I}
$$

where

$$
\mathbf{k}_{0}^{(h)}=\int_{V^{(h)}} \mathbf{B}^{(h) \mathrm{T}}(\mathbf{x}) \mathbf{D}_{0} \mathbf{B}^{(h)}(\mathbf{x}) \mathrm{d} V^{(h)}
$$

denotes the element nominal stiffness matrix, while

$$
\mathbf{k}_{i}^{(h)}=\sqrt{\lambda_{i}} \int_{V^{(h)}} \psi_{i}(\mathbf{x}) \mathbf{B}^{(h) \mathrm{T}}(\mathbf{x}) \mathbf{D}_{0} \mathbf{B}^{(h)}(\mathbf{x}) \mathrm{d} V^{(h)}
$$

represents the deviation matrix associated to the $i-$ th term of the KL-like decomposition. It is worth remarking that the interval element stiffness matrix in Eq. (17) is affected simultaneously by all the EUIs describing the uncertainty of the spatially dependent Young's modulus over the body domain.

Like in the standard FEM, the interval nodal displacement vector of the $h$-th FE, $\mathbf{d}^{(h) I}$, is related to the global nodal displacements, collected into the interval vector $\mathbf{U}^{I}$, by the following relationship: 


$$
\mathbf{d}^{(h) I}=\mathbf{L}^{(h)} \mathbf{U}^{I}
$$

where $\mathbf{L}^{(h)}$ is the connectivity matrix.

Substituting Eq. (20) in Eq. (14) and imposing the stationarity conditions of the ITPE, the following set of linear interval equations governing the equilibrium of the FE model is obtained:

$$
\mathbf{K}^{I} \mathbf{U}^{I}=\mathbf{F}
$$

In the previous equation, $\mathbf{K}^{I}$ is the interval global stiffness matrix, defined as:

$$
\mathbf{K}^{I}=\mathbf{K}_{0}+\sum_{i=1}^{M} \mathbf{K}_{i} \hat{e}_{i}^{I}
$$

where $\mathbf{K}_{0}$ denotes the global nominal stiffness matrix and $\mathbf{K}_{i}$ is the global deviation stiffness matrix associated with the $i-$ th term of the KL-like decomposition of the uncertain Young's modulus (7), given, respectively, by:

$$
\begin{aligned}
& \mathbf{K}_{0}=\sum_{h=1}^{N} \mathbf{L}^{(h) \mathrm{T}} \mathbf{k}_{0}^{(h)} \mathbf{L}^{(h)} ; \\
& \mathbf{K}_{i}=\sum_{h=1}^{N} \mathbf{L}^{(h) \mathrm{T}} \mathbf{k}_{i}^{(h)} \mathbf{L}^{(h)} .
\end{aligned}
$$

By inspection of Eq. (22), it is observed that the deviation of the interval global stiffness matrix from the nominal one is given by the superposition of the deviation matrices, $\mathbf{K}_{i}$, weighted by the corresponding EUIs, $\hat{e}_{i}^{I}$.

Finally, in Eq. (21) $\mathbf{F}$ is the global nodal force vector defined as follows:

$$
\mathbf{F}=\sum_{h=1}^{N} \mathbf{L}^{(h) \mathrm{T}} \mathbf{f}^{(h)}
$$

It is worth remarking that Eq. (21) is formally analogous to the set of linear interval equations governing the equilibrium of FE modelled structures with uncertain properties described by a 
number of discrete interval variables (see e.g., [23],[24]). Such a notable feature allows us to apply the same propagation strategies to deal either with interval field or discrete interval variable descriptions of the uncertain input. In this regard, however, it is observed that the number of independent interval variables of the model (see Eq.(22)), say $M$, is not related to the number of FEs of the selected mesh, as is customary in classical IFEMs, but it is always given by the truncation order $M$ of the KL-like decomposition. This property generally implies a considerable reduction of the computational burden of the subsequent uncertainty propagation analysis. In view of the analogy between the spatial dependency function and the autocorrelation function, the optimal truncation order $M$ may be chosen relying on the large number of studies devoted to the convergence of the KL expansion of random fields (see e.g., [39]).

\section{APPROXIMATE EXPLICIT BOUNDS OF THE RESPONSE}

The exact solution of the linear interval global equilibrium equations (21) proves to be a nontrivial task. Indeed, the set containing all the solutions of Eq. (21), obtained when the EUIs range independently between -1 and +1 , may be very complicated and its exact computation is challenging. Such a solution set can be formally defined as:

$$
\Sigma=\left\{\mathbf{U} \in \mathbb{R}^{n} \mid \mathbf{K} \mathbf{U}=\mathbf{F}, \hat{e}_{i} \in \hat{e}_{i}^{I}=[-1,+1]\right\}
$$

where $n$ is the number of degrees-of-freedom (DOFs) of the FE model; $\{S \mid P\}$ means "the set of quantities $S$ such that the proposition $P$ holds". The square interval matrix $\mathbf{K}^{I}$ is regular, that is each matrix $\mathbf{K} \in \mathbf{K}^{I}$ is non-singular [46]; this implies that the solution $\mathbf{U}^{I}$ of Eq. (21) exists for all $\mathbf{K} \in \mathbf{K}^{I}$. In the literature, several attempts have been made to develop interval versions of classical direct or iterative algorithms [16], such as Gaussian elimination or the Gauss-Seidel method. Due to the large number of interval computations involved, however, 
such algorithms are strongly affected by the dependency phenomenon [16], which leads to extremely conservative solutions for real FE models. Over the last decades, much research effort has been devoted to develop alternative solution strategies able to limit the overestimation of the interval output range, so as to enhance the application of the interval model of uncertainty in the field of engineering. In this context, some approaches, such as the ones based on Interval Rational Series Expansion (IRSE) [23] or the response surface method [24],[35], focus on the derivation of approximate explicit expressions of the solution of Eq. (21) as a function of the interval parameters. The knowledge of such expressions allows a straightforward computation of the approximate $\mathrm{LB}$ and $\mathrm{UB}$ of the interval displacement vector $\mathbf{U}^{I}$, containing the solution set $\Sigma$ in Eq. (25), which has the narrowest interval components.

\subsection{Bounds of the interval displacements}

In the present study, the bounds of the interval displacement vector $\mathbf{U}^{I}$ are evaluated by applying a response surface approach recently proposed by one of the authors for the analysis of Euler-Bernoulli beams with interval Young's modulus [35].

Let us assume that the $j$-th interval displacement component, $U_{j}^{I}$, can be approximated as the sum of the nominal value, $U_{0, j}$, plus a deviation due to the $M$ terms of the KL-like decomposition of the uncertain Young's modulus (7), say to the EUIs $\hat{e}_{i}^{I}(i=1,2, \ldots, M)$, separately taken, i.e.:

$$
U_{j}^{I}=U_{0, j}+\sum_{i=1}^{M} U_{i, j}^{I}
$$

Further, let the deviation, $U_{i, j}^{I}$, associated with the $i$-th $E U I$ be approximated by a rational function of $\hat{e}_{i}^{I}$, so that Eq. (26) can be recast as: 


$$
U_{j}^{I}=U_{0, j}+\sum_{i=1}^{M} U_{i, j}^{I}=U_{0, j}+\sum_{i=1}^{M} \frac{\hat{e}_{i}^{I}}{A_{i, j}+B_{i, j} \hat{e}_{i}^{I}} \equiv U_{j}\left(\hat{\mathbf{e}}^{I}\right)
$$

where $\hat{\mathbf{e}}^{I}=\left[\begin{array}{llll}\hat{e}_{1}^{I} & \hat{e}_{2}^{I} & \ldots & \hat{e}_{M}^{I}\end{array}\right]^{T}$ is the interval vector collecting the $M E U I \mathrm{~s} ; A_{i, j}$ and $B_{i, j}$ are $2 M$ unknown coefficients. Such coefficients can be evaluated by fitting the approximate response (27) to the exact implicit one at $2 M$ selected sampling points which define an appropriate design of experiment [47]. Within the interval framework, an effective selection of the sampling points consists of setting all the EUIs equal to zero except the $i-$ th interval which is set either to the UB or to the LB, i.e.: $\hat{e}_{i}^{I}=+1, \hat{e}_{j}^{I}=0, i \neq j=1,2, \ldots, M ; \hat{e}_{i}^{I}=-1, \hat{e}_{j}^{I}=0$, $i \neq j=1,2, \ldots, M$. Thus, the evaluation of the coefficients $A_{i, j}$ and $B_{i, j}$ requires the solution of the following $2 M+1$ sets of linear algebraic equations:

$$
\begin{aligned}
& \mathbf{U}_{0}=\mathbf{K}_{0}^{-1} \mathbf{F} ; \\
& \mathbf{U}^{(i)+}=\left(\mathbf{K}_{0}+\mathbf{K}_{i}\right)^{-1} \mathbf{F} ; \\
& \mathbf{U}^{(i)-}=\left(\mathbf{K}_{0}-\mathbf{K}_{i}\right)^{-1} \mathbf{F}, \quad i=1,2, \ldots, M
\end{aligned}
$$

where $\mathbf{U}_{0}$ denotes the nominal displacement vector; $\mathbf{U}^{(i)+}$ and $\mathbf{U}^{(i)-}$ are the deterministic displacement vectors obtained by setting all the EUIs equal to zero except the $i-$ th interval which is set to $\hat{e}_{i}^{I}=+1$ and $\hat{e}_{i}^{I}=-1$, respectively.

Once the coefficients $A_{i, j}$ and $B_{i, j}$ are known, Eq. (27) provides an approximate explicit expression of the response in terms of the EUIs, which can be exploited to evaluate the bounds of the interval displacement vector $\mathbf{U}^{I}$. For this purpose, different strategies can be applied, such as classical optimization procedures or combinatorial approaches, whose computational efficiency would be significantly enhanced by virtue of Eq. (27). In the present paper, a more efficient approach, able to provide analytical expressions of the bounds of the interval displacements, is adopted. To this aim, Eq. (27) is rewritten in the following affine form: 


$$
U_{j}^{I}=U_{0, j}+\sum_{i=1}^{M}\left(a_{0 i, j}+\Delta a_{i, j} \hat{e}_{i}^{I}\right)
$$

where

$$
\begin{aligned}
& a_{0 i, j}=\frac{\bar{U}_{i, j}+\underline{U}_{i, j}}{2} ; \\
& \Delta a_{i, j}=\frac{\bar{U}_{i, j}-\underline{U}_{i, j}}{2}
\end{aligned}
$$

are the midpoint and deviation amplitude of the $i-$ th interval deviation $U_{i, j}^{I}$ in Eq. (27), whose LB and UB, $\underline{U}_{i, j}$ and $\bar{U}_{i, j}$, are given, respectively by:

$$
\begin{aligned}
& \underline{U}_{i, j}=\min \left\{\frac{-1}{A_{i, j}-B_{i, j}}, \frac{1}{A_{i, j}+B_{i, j}}\right\} ; \\
& \bar{U}_{i, j}=\max \left\{\frac{-1}{A_{i, j}-B_{i, j}}, \frac{1}{A_{i, j}+B_{i, j}}\right\} .
\end{aligned}
$$

Based on the affine form (29) and applying the rules of the IIA via EUI, the following approximate explicit expressions of the LB and UB of the $j$-th interval displacement component are obtained:

$$
\begin{aligned}
& \underline{U}_{j}=\operatorname{mid}\left\{U_{j}^{I}\right\}-\sum_{i=1}^{M} \Delta a_{i, j} \\
& \bar{U}_{j}=\operatorname{mid}\left\{U_{j}^{I}\right\}+\sum_{i=1}^{M} \Delta a_{i, j}
\end{aligned}
$$

where

$$
\operatorname{mid}\left\{U_{j}^{I}\right\}=U_{0, j}+\sum_{i=1}^{M} a_{0 i, j}
$$

is the midpoint value. 
It is worth remarking that the proposed approach is much more computationally efficient than the vertex method [37]. Indeed, the latter evaluates the exact bounds of the response as the minimum and maximum among the solutions pertaining to all possible combinations of the endpoints of the EUIs, say $2^{M}$, if $M$ terms are retained in the KL-like expansion of the uncertain Young's modulus. This implies that the vertex method requires $2^{M}$ deterministic FE analyses against the $2 M+1$ analyses (see Eqs (28a-c)) needed to define the proposed ratio of polynomial response surface.

\subsection{Bounds for the interval stress}

Substituting the interval constitutive matrix $\mathbf{D}^{I}(\mathbf{x})$ given by Eq. (10) and the proposed response surface approximation of the interval displacements (27) into Eq. (12b), the following explicit relationship between the interval stress field within the $h$-th FE and the EUIs is obtained:

$$
\boldsymbol{\sigma}^{(h) I}(\mathbf{x})=\left[\mathbf{D}_{0}+\sum_{i=1}^{M} \mathbf{D}_{i}(\mathbf{x}) \hat{e}_{i}^{I}\right] \mathbf{B}^{(h)}(\mathbf{x}) \mathbf{L}^{(h)} \mathbf{U}\left(\hat{\mathbf{e}}^{I}\right) \equiv \boldsymbol{\sigma}^{(h)}\left(\mathbf{x} ; \hat{\mathbf{e}}^{I}\right)
$$

where $\mathbf{U}\left(\hat{\mathbf{e}}^{I}\right) \equiv \mathbf{U}^{I}$ is the vector collecting the interval displacements defined in Eq. (27).

As already observed, the interval stress is affected by uncertainty both through the interval constitutive matrix and the interval global displacements. In particular, it is noted that each EUI appears more than once in the approximate explicit expression (34) of the interval stress vector. This circumstance may lead to high overestimation of the interval stress range unless suitable approaches are adopted to predict sharp bounds. Based on the knowledge of the approximate functional dependence (34) of the stress components on the EUIs, two main strategies are herein proposed to address this issue: the first one relies on a sensitivity analysis, while the second approach is conceived as an enhancement of the classical combinatorial procedure. It is worth 
remarking that, when applying these strategies, the use of the IIA [25] allows us to keep track of uncertainties throughout calculations by means of the EUIs and thus reduce the overestimation due to the dependency phenomenon.

The proposed sensitivity-based procedure relies on the observation that, at a given position $\mathbf{x}$, the stress components are monotonic functions of the EUIs. The key idea is to perform a preliminary sensitivity analysis to predict the monotonic increasing or decreasing behaviour of the stress components as functions of each EUI. The vector collecting the sensitivities of the interval stress $\boldsymbol{\sigma}^{(h)}\left(\mathbf{x} ; \hat{\mathbf{e}}^{I}\right)$ within the $h$-th FE with respect to the $i-$ th $E U I \hat{e}_{i} \in \hat{e}_{i}^{I}=[-1,+1]$ can be evaluated analytically by direct differentiation of Eq. (34), i.e.:

$$
\mathbf{S}_{\mathbf{\sigma}^{(h), i}}(\mathbf{x})=\left.\frac{\partial \boldsymbol{\sigma}^{(h)}(\mathbf{x} ; \hat{\mathbf{e}})}{\partial \hat{e}_{i}}\right|_{\hat{\mathbf{e}}=\mathbf{0}}=\mathbf{D}_{i}(\mathbf{x}) \mathbf{B}^{(h)}(\mathbf{x}) \mathbf{L}^{(h)} \mathbf{U}_{0}+\mathbf{D}_{0} \mathbf{B}^{(h)}(\mathbf{x}) \mathbf{L}^{(h)} \mathbf{A}_{i}, \quad(i=1,2, \ldots M)
$$

where $\hat{\mathbf{e}} \in \hat{\mathbf{e}}^{I} ; \mathbf{A}_{i}$ denotes a $n$-component vector whose $j$-th component is $1 / A_{i, j}$. Eq. (35 ) provides information on the change of the stress at a prescribed position $\mathbf{x}$ within the $h-$ th FE due to a change of the $i-$ th $E U I, \hat{e}_{i}^{I}$, over the range $[-1,+1]$. Specifically, the $k-$ th interval stress component, $\sigma_{k}^{(h) I}(\mathbf{x})$, is an increasing or decreasing function of $\hat{e}_{i}^{I}$ depending on whether $s_{\sigma_{k}^{(h)}, i}(\mathbf{x})>0$ or $s_{\sigma_{k}^{(h), i}}(\mathbf{x})<0$, respectively. Based on this observation, the combinations of the endpoints of the EUIs which give the LB and UB of the $k$ - th interval stress component, $\sigma_{k}^{(h) I}(\mathbf{x})$, denoted by $\hat{e}_{h, k, i}^{(\mathrm{LB})}$ and $\hat{e}_{h, k, i}^{(\mathrm{UB})}(i=1,2, \ldots, M)$, respectively, can be determined as follows:

$$
\begin{aligned}
& \text { if } s_{\sigma_{k}^{(h), i}}(\mathbf{x})>0, \text { then } \hat{e}_{h, k, i}^{(\mathrm{UB})}=+1, \quad \hat{e}_{h, k, i}^{(\mathrm{LB})}=-1 ; \\
& \text { if } s_{\sigma_{k}^{(h), i}}(\mathbf{x})<0, \text { then } \hat{e}_{h, k, i}^{(\mathrm{UB})}=-1, \quad \hat{e}_{h, k, i}^{(\mathrm{LB})}=+1, \quad(i=1,2, \ldots, M) .
\end{aligned}
$$


The combinations $\hat{e}_{h, k, i}^{(\mathrm{LB})}$ and $\hat{e}_{h, k, i}^{(\mathrm{UB})}$ of the EUIs provided by the sensitivity analysis can be collected into the following vectors:

$$
\begin{aligned}
\hat{\mathbf{e}}_{h, k}^{(\mathrm{UB})} & =\left[\begin{array}{llll}
\hat{e}_{h, k, 1}^{(\mathrm{UB})} & \hat{e}_{h, k, 2}^{(\mathrm{UB})} & \ldots & \hat{e}_{h, k, M}^{(\mathrm{UB})}
\end{array}\right]^{\mathrm{T}} ; \\
\hat{\mathbf{e}}_{h, k}^{(\mathrm{LB})} & =\left[\begin{array}{llll}
\hat{e}_{h, k, 1}^{(\mathrm{LB})} & \hat{e}_{h, k, 2}^{(\mathrm{LB})} & \ldots & \hat{\boldsymbol{e}}_{h, k, M}^{(\mathrm{LB})}
\end{array}\right]^{\mathrm{T}} .
\end{aligned}
$$

Then, approximate explicit expressions of the LB and UB of the $k$-th interval stress component, $\sigma_{k}^{(h) I}(\mathbf{x})$, can be readily obtained by substituting the vectors $\hat{\mathbf{e}}_{h, k}^{(\mathrm{LB})}$ and $\hat{\mathbf{e}}_{h, k}^{(\mathrm{UB})}$, respectively, into Eq. (34), i.e.:

$$
\begin{aligned}
& \underline{\sigma}_{k}^{(h)}(\mathbf{x})=\sigma_{k}^{(h)}\left(\mathbf{x} ; \hat{\mathbf{e}}_{h, k}^{(\mathrm{LB})}\right) ; \\
& \bar{\sigma}_{k}^{(h)}(\mathbf{x})=\sigma_{k}^{(h)}\left(\mathbf{x} ; \hat{\mathbf{e}}_{h, k}^{(\mathrm{UB})}\right) .
\end{aligned}
$$

Alternatively, instead of using Eq. (34), two separate deterministic FE analyses can be run for the two combinations of the EUIs specified in Eqs. $(37 \mathrm{a}, \mathrm{b})$, at the expense of higher computational time.

It is worth mentioning that the sensitivity-based approach may be time-consuming since the combinations of the endpoints of the EUIs $(37 \mathrm{a}, \mathrm{b})$ need to be computed for each stress component. However, in practical engineering, often a knowledge of the extreme values of stress components at a few critical points is of interest for design purposes.

The alternative approach herein proposed for evaluating the bounds of stress components relies on the use of the ratio of polynomial response surface (34) in conjunction with the vertex method. The key idea is to evaluate the stress components pertaining to all possible combinations of the bounds of the EUIs, say $2^{M}$, by simply substituting such combinations into Eq. (34 ) rather than repeating the solution of the equilibrium equations (21). Then, following the classical combinatorial procedure, the LB and UB of each stress component are identified as the minimum and maximum among the $2^{M}$ computed stresses, respectively. The computational times are drastically reduced compared to the crude vertex method which, as already mentioned, 
requires $2^{M}$ deterministic analyses to be performed with the associated inversions of the global stiffness matrix (see Eq.(21)).

\section{IMPLEMENTATION}

The proposed IFEM, which relies on the use of the interval field model, enables the spatial dependency of uncertainties to be taken into account, leading to tremendous computational savings compared to standard IFEMs. The formulation of the method (see Section 2) retains the main steps of the deterministic FEM, such as the standard assembly procedure which yields the interval global equilibrium equations governing the discretized model. Furthermore, the proposed response surface approach for propagating the interval field exhibits a non-intrusive nature, since it just involves a sequence of deterministic FE analyses. These desirable features allow the developed IFEM to be incorporated into commercial FE software in a straightforward manner. In the present study, a computational tool consisting of a combination of User Subroutines and Python scripts is developed to integrate the proposed IFEM into the commercial code ABAQUS. A similar procedure to that described here can be adopted to incorporate the methodology in other FE codes, provided they permit the user access to routines which allow manipulation of the constitutive relationships employed in the models.

To incorporate the interval field model of Young's modulus in ABAQUS, we need to implement the interval constitutive matrix of Eq. (10), which depends on the position vector $\mathbf{x}$, and is expressed as the sum of the nominal value plus an interval deviation given by the superposition of independent contributions associated to the EUIs. This task is herein efficiently accomplished by coding either User MATerial (UMAT) or USerDefinedFieLD (USDFLD) subroutines, written in FORTRAN 77. 
The general purpose of the UMAT subroutine is to define the constitutive behavior. The UMAT subroutine is called at every integration point of the numerical integration scheme adopted by ABAQUS to evaluate element properties. This feature enables the spatial dependency of the interval Young's modulus to be taken into account by evaluating the interval constitutive matrix at the $N_{G}$ integration points of the model, i.e.

$$
\mathbf{D}^{I}\left(\mathbf{X}_{G, j}\right)=\mathbf{D}_{0}\left[1+\sum_{i=1}^{M} \sqrt{\lambda_{i}} \psi_{i}\left(\mathbf{X}_{G, j}\right) \hat{e}_{i}^{I}\right]=\mathbf{D}_{0}+\sum_{i=1}^{M} \mathbf{D}_{i}\left(\mathbf{X}_{G, j}\right) \hat{e}_{i}^{I}, \quad\left(j=1,2, \ldots, N_{G}\right)
$$

where $\mathbf{X}_{G, j}$ is the vector which contains the coordinates of the $j$-th integration point. As will be outlined in detail next, to implement the proposed response surface approach for uncertainty propagation (see Section 3), the EUIs appearing in Eq. (39) are conveniently treated as parameters which are set either to -1 or +1 within the context of the analysis.

Once the UMAT is implemented, the evaluation of the interval element stiffness matrix in Eq. (15), for any selected element type, is performed by ABAQUS through numerical integration. Repeated calls of the UMAT allow the evaluation of the interval constitutive matrix $\mathbf{D}^{I}\left(\mathbf{X}_{G, j}\right)$ at each integration point.

It is observed that Eq. (39) requires the knowledge of the eigenvalues and eigenfunctions of the spatial dependency function $\Gamma_{B}(\mathbf{x}, \xi)$, which are solutions of the Fredholm integral equation reported in Eq. (5). For regular domains and selected analytical expressions of the spatial dependency function, such as exponential, closed-form expressions of the eigenvalues and eigenfunctions are available [40]. In general, a numerical solution of the Fredholm integral equation is needed [41]. For this purpose, FE based Galerkin approaches are most often used in the literature [40]. The use of these methods leads to dense and computationally expensive matri- 
ces, especially for 2D and 3D domains [42]. Several strategies have been proposed in the literature to achieve an accurate and efficient solution of the integral eigenvalue problem in Eq. (5) (see e.g., [42], [43], [44], [45]).

Alternatively, the interval field representation of the uncertain Young's modulus based on the IIA via EUI can be introduced into the linear-elastic constitutive model of the material by coding a USDFLD subroutine, which allows the user to define spatially varying field variables (FVs). Specifically, the uncertain Young's modulus in Eq. (7) is defined so as to be proportional to an interval $F V$ which is evaluated at each integration point as:

$$
F V^{I}\left(\mathbf{X}_{G, j}\right)=1+\sum_{i=1}^{M} \sqrt{\lambda_{i}} \psi_{i}\left(\mathbf{X}_{G, j}\right) \hat{e}_{i}^{I}, \quad\left(j=1,2, \ldots, N_{G}\right)
$$

where, also in this case, the EUIs are treated as parameters which can be set either to -1 or +1 within the context of the analysis.

Based on Eq. (40), the interval constitutive matrix in Eq. (10) can be readily computed at the $N_{G}$ integration points as follows:

$$
\mathbf{D}^{I}\left(\mathbf{X}_{G, j}\right)=\mathbf{D}_{0} F V^{I}\left(\mathbf{X}_{G, j}\right), \quad\left(j=1,2, \ldots, N_{G}\right)
$$

Then, the evaluation of the interval element stiffness matrix in Eq. (15) can be performed by ABAQUS through numerical integration for any type of element. As before, eigenvalues and eigenfunctions of the spatial dependency function $\Gamma_{B}(\mathbf{x}, \xi)$ at the integration points must be specified.

Once the interval field description of the uncertain Young's modulus is incorporated into the FE formulation, coded as either UMAT or USDFLD subroutines, the analysis can take advantage of ABAQUS' pre- and post-processing interfaces and exploit the computational capabilities of the ABAQUS Standard solver. Moreover, it is worth emphasizing that, the implemented interval constitutive behavior of the material can be used in conjunction with any type of element contained in ABAQUS' libraries without writing ad hoc code for each FE type. 
The next step of the implementation of the proposed IFEM concerns the solution of the interval global equilibrium equations (21) by means of the response surface approach described in Section 3. In this regard, it is recalled that the definition of the ratio of polynomial response surface (see Eq. (27)) requires a certain number of deterministic analyses to be performed which differ from one another in the values assumed by the EUIs (see Eqs. (28a-c)). Such deterministic analyses are herein efficiently performed as parametric studies by coding Python scripts. Indeed, as already mentioned, the interval constitutive matrix, either in the context of the UMAT (see Eq. (39)) or USDFLD (see Eq. (41)), is treated as a parametric matrix with the EUIs playing the role of parameters which can take either the value -1 or +1 . The Python script to implement parametric studies in ABAQUS contains the instructions needed to generate, execute, and gather the results of multiple analyses that differ only in the values of some of the parameters herein represented by the EUIs. The Python script requires a parameterized input file, containing the geometry of the problem, boundary and load conditions, the assigned type of FE and the parametric material constitutive behavior, which is defined through a User Subroutine (UMAT or USDFLD) and depends on the values assumed by the EUIs. The assembly procedure is then automatically performed by ABAQUS, which delivers the global stiffness matrix, as well as the load and boundary conditions, to the numerical solver.

The computational framework developed in the present study provides a simple, efficient and versatile tool to analyze complex structures exhibiting non-deterministic parameters, able to enrich the formulation of well-established deterministic FEs by introducing the interval field representation of the uncertain properties.

For problems involving complex domains, large computational times may be needed for the numerical solution of Eq.(5). Thus, the crucial issue in the application of the developed computational scheme to real engineering problems is the ability to compute a large number of eigenpairs of the spatial dependency function accurately and rapidly. To this aim, numerical 
methods proposed within a probabilistic framework for the solution of the Fredholm integral equation (5) with arbitrary integral kernel over non-rectangular domains may be adopted (see e.g. [42], [43], [44], [45]).

It is worth mentioning that the proposed approach is also able to analyze problems involving multi-interval fields. In the present study, for the sake of simplicity, only Young's modulus of the material is assumed to be uncertain. Additional uncertain properties described as interval fields, such as Poisson's ratio, can be incorporated into the FE formulation by coding suitable User Subroutines. This would imply an increase of the dimensionality of uncertainty which can be handled by the proposed propagation strategy with reasonable computational costs.

Finally, it is observed that the non-intrusive implementation presented in the paper can be readily extended to the case of uncertain properties modelled as random fields. Indeed, as outlined in Ref. [29], the interval field model based on the IIA via EUI is formally analogous to the classical KL expansion of a random field consisting of a superposition of deterministic spatial functions with corresponding random coefficients [40]. Within the probabilistic framework, a set of uncorrelated standard random variables plays the same role of the EUIs. The propagation of random fields is more time consuming than the one of interval fields, especially when higher-order response statistics are desired. The probabilistic characterization of the response may be carried out by using the proposed ratio of polynomial response surface in conjunction with Monte Carlo simulation [24]. As known, however, the computational efficiency of sampling-based procedures rapidly worsens as the truncation order of the KL expansion increases since samples of a large number of random variables need to be generated. Conversely, the bounds of the interval response can be efficiently evaluated even for large truncation orders $M$ of the KL-like decomposition of the interval field. 


\section{NUMERICAL APPLICATIONS}

The proposed IFEM implemented in ABAQUS is applied to analyze two square plates under different loading and boundary conditions. In both cases, the constitutive behaviour of the material is assumed to be linear-elastic isotropic with uncertain Young's modulus modelled as an interval field based on the IIA (see Eq.(7)), i.e.:

$$
E^{I}(x, y)=E_{0}\left[1+B^{I}(x, y)\right]=E_{0}\left[1+\sum_{i=1}^{M} \sqrt{\lambda_{i}} \psi_{i}(x, y) \hat{e}_{i}^{I}\right]
$$

where $x$ and $y$ are the Cartesian coordinates of a generic point of the 2D domain of the plates.

The spatial dependency function (see Eq.(3)) characterizing the interval field $E^{I}(x, y)$ in Eq. (42) is assumed to have the following exponential form:

$$
\Gamma_{B}(x, y ; \xi, \eta)=C_{B}^{2} \exp \left(-\frac{|x-\xi|}{l_{B x}}-\frac{|y-\eta|}{l_{B y}}\right)
$$

where $(x, y)$ and $(\xi, \eta)$ are the Cartesian coordinates of two different points of the 2D domain. The parameter $C_{B}$ may be regarded as the non-probabilistic counterpart to the standard deviation in random field theory [6], since it affects the deviation amplitude of the interval field and thus the degree of uncertainty. Similarly, $l_{B x}$ and $l_{B y}$ may be viewed as the analogue of the correlation lengths since they rule the spatial dependency of the uncertain property along the $x$ - and $y$-directions. Without loss of generality, it is herein assumed that $l_{B x}=l_{B y}=l_{B}$. Notice that, if $l_{B} \rightarrow \infty$, the spatial dependency function in Eq. (43) approaches the value $C_{B}^{2}$, and the dimensionless interval function $B^{I}(x, y)$ in Eq. (42) reduces to a symmetric interval variable, i.e. $B^{I}(x, y) \equiv b^{I}=b \hat{e}^{I}$ with deviation amplitude $b=C_{B}$. This circumstance implies the TSD of the uncertain Young's modulus which, indeed, turns out to be described by a single interval 
variable over the whole domain i.e. $E^{I}(x, y) \equiv E^{I}=E_{0}\left(1+b \hat{e}^{I}\right)$. At the opposite extreme, as $l_{B} \rightarrow 0$, the TSI of the uncertain material property is achieved. In this case, Young's moduli of the FEs of the selected mesh are described by independent interval variables (see e.g., $[23],[24])$.

It is worth mentioning that, for regular domains, such as those employed in the square plates analyzed here, the eigenvalues and eigenfunctions of the exponential function in Eq. (43) can be evaluated in analytical form [40].

The accuracy of the proposed IFEM is assessed by performing appropriate comparisons with the bounds of the response provided by the vertex method, which requires $2^{M}$ deterministic analyses, $M$ being the truncation order of the KL-like expansion of the uncertain Young's modulus in Eq. (42).

\subsection{Plate under uniform traction with interval Young's modulus}

The first application concerns a typical plane stress problem, i.e. a square plate clamped along one edge and subjected to a uniformly distributed traction along the opposite edge (Figure 1). The material is assumed to have uncertain Young's modulus described by the interval field in Eq. (42). The following data are considered: width and thickness of the plate $L=0.1 \mathrm{~m}$ and $t=0.001 \mathrm{~m}$, respectively; nominal Young's modulus $E_{0}=210 \mathrm{GPa}$; Poisson's ratio $v=0.3$; traction $p=10 \mathrm{MPa}$. The plate is discretized into $N=16$ plane stress four-node elements. A complete Gauss quadrature integration rule is adopted. The interval nodal displacements in the load direction, $U_{j y}^{I},(j=1,2, \ldots, 20)$, are selected as response quantities of interest.

Both UMAT and USDFLD subroutines have been coded to incorporate the interval field representation of Young's modulus into the formulation. 
The truncation order $M$ of the KL-like decomposition is selected by analyzing the rate of convergence of the proposed response surface approximation (see Eq. (27)). Specifically, attention is focused on the LB and UB of the interval displacement of node 20 in the load direction, $U_{20 y}^{I}$. In Figure 2, such bounds are plotted versus the truncation order $M$, for $C_{B}=0.05$ and different values of the parameter $l_{B}$. By inspection of Figure 2, it can be inferred that, as the parameter $l_{B}$ increases, the series converges more quickly, so that a smaller number of terms is required to represent the response surface in Eq. (27) and, therefore, the interval Young's modulus in Eq. (42). To ensure a reasonable trade-off between accuracy and computational efficiency, $M=10$ terms are retained for all values of the parameter $l_{B}$ herein considered. Alternatively, the optimal truncation order $M$ may be selected referring to suitable error measures similar to those introduced in the literature for assessing the accuracy of the truncated KL expansion of random fields (see e.g., [42],[44]). For instance, in view of the analogy between the parameter $C_{B}$ in Eq. (43) and the standard deviation, global error measures related to the variance of the random field may be translated to the interval field. Furthermore, the number of series terms may be significantly reduced by modifying the exponentially decaying spatial dependency function to remove the non-differentiability at the origin [48].

In order to highlight the main features of the 2D interval field representing the uncertain Young's modulus (42) over the plate domain, Figure 3 shows the LB function, $\underline{E}(x, y)$, the UB function, $\bar{E}(x, y)$, (see Eqs. (8a,b)), and two typical samples, $E^{(1)}(x, y)$ and $E^{(2)}(x, y)$, for $C_{B}=0.05$ and two different values of the parameter $l_{B}, l_{B}=0.5 L$ and $l_{B}=5 L$. Specifically, the samples $E^{(1)}(x, y)$ and $E^{(2)}(x, y)$ are obtained from Eq.(7) setting $\hat{e}_{i}^{I}=1, i=1,3,5$, $\hat{e}_{j}^{I}=-1, j \neq i$ and $\hat{e}_{i}^{I}=-1, i=1,5,7,8,10, \hat{e}_{j}^{I}=1, j \neq i$, respectively. As expected, the results are enclosed by the LB and UB functions. Furthermore, it is observed that, as the value of the 
parameter $l_{B}$ increases, the pattern of the interval field realizations consistently becomes more regular. Indeed, as already mentioned, when $l_{B} \rightarrow \infty$, the interval field reduces to a single interval variable over the plate domain, that is the condition of TSD of the interval Young's modulus is approached.

For validation purposes, the proposed bounds of the selected interval nodal displacements, $U_{j y}^{I},(j=1,2, \ldots, 20)$, are contrasted in Figure 4 with the ones obtained by means of the vertex method, for $l_{B}=L$ and two different values of the parameter $C_{B}$ which describes the degree of uncertainty, $C_{B}=0.05$ and $C_{B}=0.1$. It can be noticed that the estimates of the LB and UB of the nodal displacements provided by the proposed IFEM are in very good agreement with the ones yielded by the vertex method, even for relatively high degrees of uncertainty. Furthermore, as expected, the region of the interval displacements widens when larger values of $C_{B}$ are considered.

A further insight into the propagation of Young's modulus uncertainty can be gained by evaluating the so-called coefficient of interval uncertainty (c.i.u.), which provides a measure of the dispersion of the response quantity of interest around its midpoint value. The c.i.u. of the nodal displacements in the load direction, $U_{j y}^{I},(j=1,2, \ldots, 20)$, is defined as follows:

$$
\text { c.i.u. }\left[U_{j y}^{I}\right]=\frac{\Delta U_{j y}}{\left|\operatorname{mid}\left\{U_{j y}^{I}\right\}\right|}=\frac{\bar{U}_{j y}-\underline{U}_{j y}}{\left|\bar{U}_{j y}+\underline{U}_{j y}\right|} \text {. }
$$

Figure 5 shows the comparison between the c.i.u. of the nodal displacements $U_{j y}^{I}$ provided by the proposed method and the vertex method for $l_{B}=L$ and two different values of the parameter $C_{B}, C_{B}=0.05$ and $C_{B}=0.1$. Besides the accuracy of the proposed method, it is observed that the dispersion of the response around the midpoint value consistently increases with the parameter $C_{B}$. Furthermore, it can be noticed that the uncertain Young's modulus has a 
different influence on the selected degrees of freedom, with the interval displacements of nodes 1 and $5, U_{1 y}^{I}$ and $U_{5 y}^{I}$, exhibiting the largest dispersion around their midpoint value. These nodes are at the edges of the plate and experience less constraint than nodes within the bulk of the plate away from a free surface.

Attention is now focused on the influence of the spatial dependency of the interval Young's modulus on the response. To this aim, in Figure 6, the proposed c.i.u. of the displacement of node 20 in the load direction, $U_{20 y}^{I}$, versus the ratio $l_{B} / L$ is plotted $\left(C_{B}=0.05\right)$. Notice that the dispersion of the selected response quantity around its nominal value is significantly affected by the parameter $l_{B}$ governing the spatial dependency of the uncertain material property. In particular, the c.i.u. of the interval displacement $U_{20 y}^{I}$ is generally greater than the value c.i.u. $\left[U_{20 y}^{I}\right] \equiv C_{B}=0.05$ pertaining to the case of TSD $\left(l_{B} \rightarrow \infty\right)$ of the interval Young's modulus.

The influence of the spatial dependency of the uncertain material property on the response of the plate is further scrutinized in Figure 7 where the LB and UB of the interval displacement of node 20 in the load direction, $U_{20 y}^{I}$, versus the ratio $l_{B} / L$ are plotted $\left(C_{B}=0.05\right)$. Specifically, the bounds provided by the proposed IFEM, assuming the interval field representation of Young's modulus, are compared with the bounds pertaining to the extreme assumptions of TSD $\left(l_{B} \rightarrow \infty\right)$ and TSI $\left(l_{B} \rightarrow 0\right)$ of the uncertain material property over the plate domain. The latter are obtained by applying the vertex method. As shown in Figure 7, the interval field model yields a region of the interval displacement $U_{20 y}^{I}$ which is wider than the one predicted under the assumption of $\operatorname{TSD}\left(l_{B} \rightarrow \infty\right)$, whatever value of the parameter $l_{B}$ is selected. This implies that, in spite of its simplicity, the representation of Young's modulus as a single interval varia- 
ble over the whole domain may lead to serious underestimation of response variability. Conversely, under the assumption of TSI $\left(l_{B} \rightarrow 0\right)$, the width of the region of the interval displacement $U_{20 y}^{I}$ may be underestimated or overestimated depending on the value of the parameter $l_{B}$. It follows, that the spatial dependency of interval uncertainties plays a crucial role in order to obtain a reliable prediction of response variability.

As outlined in Section 3, at a prescribed location, the response of the plate is a monotonic function of the EUIs appearing in the interval field representation (42) of Young's modulus. It follows that, in general, the bounds of the interval displacements correspond to different combinations of the extreme values of the EUIs and therefore to different realizations of the uncertain Young's modulus (see Eq. (37a,b)). Figure 8 displays the samples of the interval Young's modulus $\left(C_{B}=0.05, l_{B}=0.5 L\right), E^{(\mathrm{LB})}(x, y)$ and $E^{(\mathrm{UB})}(x, y)$, which yield the LB and UB, $\underline{U}_{20 y}$ and $\bar{U}_{20 y}$, of the interval displacement of node 20 in the load direction, $U_{20 y}^{I}$ (see Figure 1). The spatial variability of $E^{(\mathrm{LB})}(x, y)$ consistently entails larger values of Young's modulus close to node 20 and lower values far from the node, whereas an opposite pattern is exhibited by the sample $E^{(\mathrm{UB})}(x, y)$. Figure 9 shows the contour plots of the displacement of the plate along the load direction corresponding to the samples of the uncertain Young's modulus shown in Figure 8, where the bounds, $\underline{U}_{20 y}$ and $\bar{U}_{20 y}$, of the interval displacement $U_{20 y}^{I}$ are achieved.

Finally, the capability of the proposed IFEM to yield accurate predictions of the interval stress over the plate is investigated. As outlined in Section 3, the bounds of the interval stress can be evaluated combining the response surface approximation (34) either with sensitivity analysis or with a combinatorial procedure. Figure 10 shows the comparison between the LB and UB of the stress component in the load direction at the Gauss points of FE 12 and FE 16 (see Figure 1) provided by the proposed method and the vertex method for $l_{B}=L$ and $C_{B}=0.05$ 
. Notice that the proposed stress bounds, obtained by applying the response surface approximation (34) in conjunction with the combinatorial procedure, are very close to the ones provided by the vertex method. Very accurate estimates, herein omitted for conciseness, are also provided by the sensitivity-based procedure described in Section 3. For comparison purposes, Figure 10 also displays the nominal stress component (i.e. for $E(x, y)=E_{0}$ ) in the load direction at the Gauss points of FE 12 and FE 16 which turns out to be enclosed by the LBs and UBs predicted in the presence of spatially varying uncertainty. It has to be mentioned that, for the analyzed plate, if the uncertain Young's modulus were assumed TSD $\left(l_{B} \rightarrow \infty\right)$, the stress would not be affected by uncertainty and the nominal stress would be obtained for any value of the TSD Young's modulus. This implies that spatial variability of the uncertain material property significantly affects the stress distribution over the plate.

It is worth emphasizing that, for the selected case study, the vertex method and the proposed procedure require $2^{M}=2^{10}$ and $2 M+1=21$ deterministic analyses, respectively. Thus, the IFEM developed in the present study enables the prediction of very accurate estimates of the bounds of both interval displacements and stresses, which require much lower computational effort than the classical combinatorial procedure. In this regard, it is recalled that the deterministic analyses needed to apply either the proposed response surface approach or the vertex method are efficiently performed exploiting ABAQUS' computational power.

\subsection{Plate under uniformly distributed transverse load with interval Young's modulus}

As a second numerical application, a simply-supported square plate under downward uniformly distributed transverse load $q$ (see Figure 11a) is considered. The constituent material is assumed to be linear-elastic isotropic with Poisson's ratio $v=0.25$ and uncertain Young's modulus described by the 2D interval field (42) with exponential spatial dependency function (43). 
The ratio between the width and thickness of the plate is assumed to be $L / t=100$. The $z$-axis is taken positive downward from the $x-y$ plane (see Figure 11a). The plate is discretized into $N=800$ three noded triangular thin shell elements. Complete Gauss quadrature integration is adopted. The normalized interval displacement of the central node A (see Figure 11b) in the load direction, $\tilde{U}_{A z}^{I}=\left(E_{0} t^{3} 10^{2} / q L^{4}\right) U_{A z}^{I}$, and the normalized interval rotation around the $x-$ axis of node B (see Figure 10b), $\tilde{\varphi}_{B x}^{I}=\left(E_{0} t^{3} / q L^{3}\right) \varphi_{B x}^{I}$, are selected as response quantities of interest.

Figure 12 shows the proposed estimates of the UB and LB of the normalized interval displacement of node A, $\tilde{U}_{A z}^{I}$, versus the truncation order $M$ of the KL-like decomposition of the interval Young's modulus (42) for different values of the parameter $l_{B}$. By examining the convergence rate of the bounds of $\tilde{U}_{A z}^{I}$, the first $M=10$ terms are retained for all values of the parameter $l_{B}$ herein considered.

Table 1 and Table 2 list the bounds of the response quantities of interest, $\tilde{U}_{A z}^{I}$ and $\tilde{\varphi}_{B x}^{I}$, respectively, provided by the proposed method and the vertex method along with the associated absolute percentage errors, obtained assuming $l_{B}=0.5 \mathrm{~L}$ and two different values of the parameter $C_{B}, C_{B}=0.05$ and $C_{B}=0.1$. Notice that the proposed IFEM yields very accurate estimates of the bounds of the selected normalized nodal displacement and rotation even for relatively high degrees of uncertainty.

Figures 13 and 14 display the samples of the normalized interval Young's modulus $\left(C_{B}=0.05, l_{B}=0.5 L\right), E^{(\mathrm{LB}), i}(x, y) / E_{0}$ and $E^{(\mathrm{UB}), i}(x, y) / E_{0}(i=1,2)$, which yield the $\mathrm{LB}$ and UB of the normalized interval deflection of node A. Due to the symmetry of the analyzed plate, each bound of the central node deflection can be achieved considering two different realizations of Young's modulus. Furthermore, the spatial variability of the samples $E^{(\mathrm{LB}), i}(x, y) / E_{0}$ 
$(i=1,2)$ consistently implies larger values of Young's modulus close to node A, whereas the samples $E^{(\mathrm{UB}), i}(x, y) / E_{0}(i=1,2)$ exhibit an opposite pattern.

In order to investigate the influence of the spatial dependency of the uncertain property on the response, the $\mathrm{LB}$ and $\mathrm{UB}$ of the interval response quantities of interest, $\tilde{U}_{A z}^{I}$ and $\tilde{\varphi}_{B x}^{I}$, versus the ratio $l_{B} / L$ are plotted in Figure 15 for $C_{B}=0.05$. In particular, the proposed bounds are compared with the ones obtained under the extreme assumptions of TSD $\left(l_{B} \rightarrow \infty\right)$ and TSI ( $l_{B} \rightarrow 0$ ) of the uncertain Young's modulus over the plate domain. Notice that, for the present case-study, the TSI assumption $\left(l_{B} \rightarrow 0\right)$ implies the introduction of $N=800$ interval variables to describe the uncertain Young's moduli of the FEs of the adopted mesh. The vertex method is obviously unfeasible since it would require $2^{800}$ deterministic analyses. For this reason, the bounds of the response pertaining to the TSI condition $\left(l_{B} \rightarrow 0\right)$ are herein evaluated by applying a sensitivity-based procedure [23]. Such a procedure highlights that, for the problem under consideration, the bounds of the normalized interval deflection $\tilde{U}_{A z}^{I}$ are achieved assuming the so-called trivial combinations of the uncertain parameters, that is setting all the interval Young's moduli either to their LB or their UB. As shown in Figure 15a, this entails that identical bounds of the normalized interval deflection $\tilde{U}_{A z}^{I}$ are obtained under the extreme assumptions of TSD $\left(l_{B} \rightarrow \infty\right)$ and TSI $\left(l_{B} \rightarrow 0\right)$. Such bounds generally enclose the ones obtained adopting the interval field model. Furthermore, it is observed that, as the ratio $l_{B} / L$ increases, the bounds of the normalized interval deflection $\tilde{U}_{A z}^{I}$ pertaining to the interval field model consistently approach the LB and UB obtained assuming TSD $\left(l_{B} \rightarrow \infty\right)$ or TSI $\left(l_{B} \rightarrow 0\right)$ of the uncertain Young's modulus. Figure 15b shows that, except for small values of the ratio $l_{B} / L$, the region of the normalized interval rotation $\tilde{\varphi}_{B x}^{I}$ provided by the proposed IFEM is wider than the ones 
obtained under the assumptions of TSD $\left(l_{B} \rightarrow \infty\right)$ and TSI $\left(l_{B} \rightarrow 0\right)$, which therefore entail underestimation of response variability. Furthermore, it is worth mentioning that, in the case of TSI $\left(l_{B} \rightarrow 0\right.$ ), the bounds of $\tilde{\varphi}_{B x}^{I}$ do not correspond to the trivial combinations. Though very close, such bounds actually are different from the ones obtained under the assumption of TSD $\left(l_{B} \rightarrow \infty\right)$.

Further information on the influence of spatial dependency can be deduced from Figure 16, where the proposed c.i.u. of the normalized interval displacement $\tilde{U}_{A z}^{I}$ and rotation $\tilde{\varphi}_{B x}^{I}$ versus the ratio $l_{B} / L$ are plotted $\left(C_{B}=0.05\right)$. As expected, the dispersion of both the selected response quantities around the midpoint value is significantly affected by the parameter $l_{B}$. In particular, it is observed that the c.i.u. of the normalized interval displacement $\tilde{U}_{A z}^{I}$ is smaller than the value c.i.u $\left[\tilde{U}_{A z}^{I}\right] \equiv C_{B}=0.05$ pertaining to the case of $\operatorname{TSD}\left(l_{B} \rightarrow \infty\right)$. Conversely, the plot of the c.i.u. of the normalized interval rotation $\tilde{\varphi}_{B x}^{I}$ (Figure 16b) shows that, except for small values of the ratio $l_{B} / L$, the dispersion of $\tilde{\varphi}_{B x}^{I}$ around the midpoint value is greater than the one obtained under the assumption of $\operatorname{TSD}\left(l_{B} \rightarrow \infty\right)$, i.e. c.i.u[ $\left[\tilde{\varphi}_{B x}^{I}\right]>C_{B}=0.05$. Also for this case-study, numerical results demonstrate that the spatial dependency of interval uncertainty may significantly affect response variability.

Table 3 and Table 4 list the LB and UB of the normalized stress component $\tilde{\sigma}_{x x}^{I}=\sigma_{x x}^{I}(h / L)^{2} / q$ evaluated at the Gauss points of FE 419 (see Figure 9 b) by applying the vertex method and the proposed response surface approximation (34) in conjunction with sensitivity analysis, for $l_{B}=0.5 \mathrm{~L}$ and two different values of $C_{B}, C_{B}=0.05$ and $C_{B}=0.1$, respectively. It is observed that the proposed IFEM provides accurate estimates of the interval stress 
components even when the degree of uncertainty increases. Very accurate results, herein omitted for conciseness, are also obtained by using the proposed response surface approximation (34) of the stress components in conjunction with the combinatorial procedure, as described in Section 3. It is worth mentioning that, also for this case study, if the uncertain Young's modulus were assumed TSD $\left(l_{B} \rightarrow \infty\right)$, the stress would not be affected by uncertainty and the nominal stress would be obtained for any value of the TSD Young's modulus. In particular, the maximum value of the normalized nominal stress $\tilde{\sigma}_{x x}^{I}=\sigma_{x x}^{I}(h / L)^{2} / q$ over the plate, which is reached at Gauss point 2 of FE 419 is 0.2760 . By inspection of Tables 3 and 4 it is inferred that this value is smaller than the UB predicted by using the interval field model. This result demonstrates the significant influence of spatial variability of the uncertain material property on the stress distribution.

Finally, Figure 17 displays the realizations of the normalized interval Young's modulus ( $\left.C_{B}=0.05, l_{B}=0.5 L\right), E^{(\mathrm{LB})}(x, y) / E_{0}$ and $E^{(\mathrm{UB})}(x, y) / E_{0}$, which yield the LB and UB of the normalized interval stress $\tilde{\sigma}_{x x}^{I}=\sigma_{x x}^{I}(h / L)^{2} / q$ at the integration point 2 of FE 419 listed in Table 3. As expected, the spatial variability of the sample $E^{(\mathrm{LB})}(x, y) / E_{0}$ is such that Young's modulus takes smaller values close to FE 419 (see Figure 11), while an opposite pattern is exhibited by the sample $E^{(\mathrm{UB})}(x, y) / E_{0}$.

\section{CONCLUSIONS}

An interval finite element method (IFEM) for the analysis of structures made of linear-elastic isotropic material with spatially varying uncertain properties has been presented. Without loss of generality, only Young's modulus of the material has been assumed to be uncertain. The 
inherent spatial dependency of the uncertain material property has been taken into account resorting to a recently proposed interval field model based on the so-called improved interval analysis via extra unitary interval (IIA via EUI). The propagation of the interval field has been performed by an efficient response surface approach which yields the bounds of both displacements and stresses in approximate explicit form. Relying on its non-intrusive nature, the developed IFEM has been integrated into the commercial FE software ABAQUS by coding suitable User Subroutines and Phyton Scripts. To the authors' knowledge, this is the first research effort in the literature to incorporate spatially varying uncertainties modelled as interval fields into a commercial FE software. The interaction with ABAQUS allows the analyst to handle, in principle, arbitrarily complex engineering problems, by exploiting the computing power of the FE solver as well as pre- and post-processing interfaces. In particular, the modelling phase may involve arbitrary types of FE belonging to FE packages libraries whose constitutive behaviour incorporates the interval field description of Young's modulus once suitable User Subroutines are coded. Thus, the analyst does not need to write a new FE code for any specific problem or any type of FE used in the modelling phase.

The main features of the proposed IFEM may be summarized as follows: $i$ ) unlike the traditional IFEMs, it takes into account the spatial dependency of the uncertain material property; ii) the dimensionality of uncertainty is drastically reduced since it does not depend on the number of FEs of the adopted mesh; iii) very accurate approximate explicit expressions of the bounds of both displacements and stresses are obtained by performing just a few deterministic analyses, even in the presence of relatively high degrees of uncertainty; $i v$ ) the computational efficiency is greatly enhanced compared to the classical combinatorial procedure; $v$ ) the implementation into the commercial FE software ABAQUS yields a very powerful and versatile tool for the analysis of complex structural systems with spatially varying properties modelled as interval fields. 
Potential extensions of the developed computational tool to the analysis of nonlinear and dynamic problems are also envisaged. Indeed, spatially varying interval uncertainties affecting the mass, stiffness and damping matrices can be readily incorporated into commercial FE software by coding suitable UMAT or USDFLD subroutines. Then, efficient propagation strategies able to exploit the potential of the FE solver need to be developed.

Acknowledgments: The authors are grateful to the three anonymous reviewers for their valuable suggestions on an earlier version of this paper.

\section{REFERENCES}

[1] Ayyub BM, Klir GJ. Uncertainty modeling and analysis in engineering and the sciences. Boca Raton: Chapman \& Hall/CRC, Taylor \& Francis Group; 2006.

[2] Corotis RB. An overview of uncertainty concepts related to mechanical and civil engineering. ASCE-ASME J. Risk Uncertainty Eng Syst, Part B: Mech Eng 2015; 1(4): 040801.

[3] Kleiber M, Hien TD. The Stochastic Finite Element Method. Chichester: John Wiley \& Sons; 1992.

[4] Stefanou G. The stochastic finite element method: Past, present and future. Comput Methods Appl Mech Eng 2009; 198: 1031-1051.

[5] Reh S, Beley J-D, Mukherjee S, Khor EH. Probabilistic finite element analysis using ANSYS. Struct Saf 2006; 28: 17-43.

[6] Vanmarcke E. Random Fields: Analysis and Synthesis. Revised and Expanded New Edition. World Scientific; 2010.

[7] Keese A. A review of recent developments in the numerical solution of stochastic partial differential equations (Stochastic Finite Elements). Internal Working Report 2003-06, Technical University, Braunschweig, Germany,2003. 
[8] Schenk CA, Schuëller GI. Uncertainty assessment of large finite element systems. LN APP C M, vol. 24, Springer; 2005.

[9] Sachdeva SK, Nair PB, Keane AJ. On using deterministic FEA software to solve problems in stochastic structural mechanics. Comput. Struct 2007; 85: 277-290.

[10]Der Kiureghian A, Haukaas T, Fujimura K. Structural reliability software at the University of California, Berkeley. Struct Saf 2006; 28: 44-67.

[11] Shang S, Yun GJ. Stochastic finite element with material uncertainties: Implementation in a general purpose simulation program. Finite Elem Anal Des 2013; 64: 65-78.

[12] Moens D, Vandepitte D. A survey of non-probabilistic uncertainty treatment in finite element analysis. Comput Methods Appl Mech Eng 2005; 194: 1527-1555.

[13] Moens D, Hanss M. Non-probabilistic finite element analysis for parametric uncertainty treatment in applied mechanics: Recent advances. Finite Elem Anal Des 2011; 47: 4-16.

[14] Moore RE. Interval Analysis Englewood Cliffs: Prentice-Hall; 1966.

[15]Ben-Haim Y, Elishakoff I. Convex Models of Uncertainty in Applied Mechanics. Amsterdam: Elsevier; 1990.

[16] Moore RE, Kearfott RB, Cloud MJ. Introduction to Interval Analysis. Philadelphia: SIAM; 2009.

[17]Zadeh L. Fuzzy sets, Inform Contr 1965; 8: 338-353.

[18]Köylüoğlu HU, Elishakoff I. A comparison of stochastic and interval finite elements applied to shear frames with uncertain stiffness properties. Comput Struct 1998; 67: 91-98.

[19] R.L. Muhanna, R.L. Mullen, Uncertainty in mechanics problems-interval-based approach, J. Eng. Mech. ASCE 127 (2001) 557-566. 
[20] Degrauwe D, Lombaert G, De Roeck G. Improving interval analysis in finite element calculations by means of affine arithmetic. Comput Struct 2010; 88: 247-254.

[21] Tangaramvong S, Wu D, Gao W, Tin-Loi F. Response bounds of elastic structures in the presence of interval uncertainties. J Struct Eng 2015; 141(12): 04015046

[22] Muhanna RL, Mullen RL, Rama Rao MV. Nonlinear finite element analysis of frames under interval material and load uncertainty. ASCE-ASME J. Risk Uncertainty Eng Syst, Part B: Mech Eng 2015; 1(4): 041003.

[23] Sofi A, E. Romeo E. A novel Interval Finite Element Method based on the improved interval analysis. Comput Methods Appl Mech Eng 2016; 311: 671-697.

[24] Sofi A, E. Romeo E. A unified response surface framework for the interval and stochastic finite element analysis of structures with uncertain parameters. Probab Eng Mech 2018; 54: 25-36.

[25] Muscolino G, Sofi A. Stochastic analysis of structures with uncertain-but-bounded parameters via improved interval analysis. Probab Eng Mech 2012; 28: 152-163.

[26] Moens D, De Munck M, Desmet W, Vandepitte D. Numerical dynamic analysis of uncertain mechanical structures based on interval fields, IUTAM Symposium on the Vibration Analysis of Structures with Uncertainties (A.K. Belyaev, R.S. Langley eds.) Springer, Dordrecht, 2011: 71-83.

[27] Verhaeghe W, Desmet W, Vandepitte D, Moens D. Interval fields to represent uncertainty on the output side of a static FE analysis. Comput Methods Appl Mech Eng 2013; 260: 5062.

[28] Imholz M, Faes M, Cerneels J, Vandepitte D, Moens D. On the Comparison of Two Novel Interval Field Formulations for the Representation of Spatial Uncertainty. In: Proceedings of the 7th International Workshop on Reliable Engineering Computing (REC2016), Eds. 
Steffen Freitag, Rafi L. Muhanna, Robert L. Mullen, June 15-17, 2016, Ruhr University Bochum, Germany, pp. 367-378.

[29] Muscolino G, Sofi A, Zingales M. One-dimensional heterogeneous solids with uncertain elastic modulus in presence of long-range interactions: interval versus stochastic analysis. Comput Struct 2013; 122: 217-229.

[30] Sofi A, Muscolino G. Static analysis of Euler-Bernoulli beams with interval Young's modulus. Comput Struct 2015; 156: 72-82.

[31] Sofi A, Muscolino G, Elishakoff I. Static response bounds of Timoshenko beams with spatially varying interval uncertainties. Acta Mech 2015; 226(11): 3737-3748.

[32]Faes M, Moens D. Identification and quantification of spatial interval uncertainty in numerical models. Comput Struct 2017; 192: 16-33.

[33] M. Faes, J. Cerneels, D. Vandepitte, D. Moens, Identification and quantification of multivariate interval uncertainty in finite element models. Comput Methods Appl Mech Eng 2017; 315: 896-920.

[34] Wu D, Gao W. Uncertain static plane stress analysis with interval fields. Int J Numer Methods Eng 2016; 110: 1201-1300.

[35] Sofi A. Euler-Bernoulli interval finite element with spatially varying uncertain properties. Acta Mech 2017; 228: 3771-3787.

[36] Wu D, Gao W. Hybrid uncertain static analysis with random and interval fields. Comput Methods Appl Mech Eng 2017; 315: 222-246.

[37]Dong W, Shah H. Vertex method for computing functions of fuzzy variables. Fuzzy Sets Syst 1987; 24: 65-78.

[38]Dassault Systèmes SIMULIA Corp. Abaqus 6.14: Abaqus Analysis User's Guide. Providence, RI, USA, 2014. 
[39]Huang SP, Quek ST, Phoon KK, Convergence study of the truncated Karhunen-Loève expansion for simulation of stochastic processes. Int J Numer Methods Eng 2001; 52 (9): $1029-1043$.

[40] Ghanem RG, Spanos PD. Stochastic Finite Elements: A Spectral Approach. New York: Springer-Verlag; 1991.

[41] Atkinson KE. The Numerical Solution of Integral Equations of the Second Kind. Cambridge University Press, 1997.

[42] Allaix DL, Carbone VI, Karhunen-Loève decomposition of random fields based on a hierarchical matrix approach. Int J Numer Methods Eng 2013; 94:1015-1036.

[43] Phoon KK, Huang SP, Quek ST. Implementation of Karhunen-Loève expansion for simulation using a wavelet-Galerkin scheme. Probab Eng Mech 2002; 17: 293-303.

[44]Betz W, Papaioannou I, Straub D. Numerical methods for the discretization of random fields by means of the Karhunen-Loève expansion. Comput Methods Appl Mech Eng 2014; 271: 109-129.

[45] Pranesh S, Ghosh D. Faster computation of the Karhunen-Loève expansion using its domain independence property. Comput Methods Appl Mech Eng 2015; 285: 125-145.

[46] Rohn J. Interval solution of linear interval equations. Appl Math 1990; 35: 220-224.

[47] Bucher C. Computational Analysis of Randomness in Structural Mechanics. London: Taylor \& Francis Group; 2009.

[48] Spanos PD, Beer M, Red-Horse J. Karhunen-Loève expansion of stochastic processes with a modified exponential covariance kernel. J Eng Mech 2007; 133 (7): 773-779. 


\section{Figure captions}

Figure 1. Square plate under uniformly distributed traction with uncertain Young's modulus.

Figure 2. Proposed a) UB and b) LB of the interval displacement of node 20 in the load direction of the plate under uniform traction versus the number $M$ of terms of the KL-like decomposition for different values of the parameter $l_{B}$.

Figure 3. LB, UB and two typical samples, $E^{(1)}(x, y)$ and $E^{(2)}(x, y)$, of the interval Young's modulus field over the plate domain for $C_{B}=0.05:$ a) $l_{B}=0.5 \mathrm{~L}$ and b) $l_{B}=5 \mathrm{~L}$

Figure 4. Bounds of the interval nodal displacements in the load direction of the plate under uniform traction: comparison between the estimates provided by the proposed method and the vertex method for $l_{B}=L$, a) $C_{B}=0.05$ and b) $C_{B}=0.1$.

Figure 5. Coefficient of interval uncertainty of the nodal displacements in the load direction of the plate under uniform traction: comparison between the estimates provided by the proposed method and the vertex method for $l_{B}=L$, and two values of $C_{B}$, namely $C_{B}=0.05$ and $C_{B}=0.1$.

Figure 6. Proposed coefficient of interval uncertainty of the displacement of node 20 in the load direction of the plate under uniform traction versus the ratio $l_{B} / L\left(C_{B}=0.05\right)$.

Figure 7. Bounds of the interval displacement of node 20 in the load direction of the plate under uniform traction versus the ratio $l_{B} / L\left(C_{B}=0.05\right)$ : comparison between the proposed estimates and the ones obtained under the assumption of TSI and TSD of the interval Young's modulus. 
Figure 8. Samples of the interval Young's modulus $\left(C_{B}=0.05, l_{B}=L\right)$ which yield the a) LB and b) UB of the interval displacement along the $y$-direction of node 20 of the plate under uniform traction.

Figure 9. Contour plot of the displacement of the plate in the $y$-direction corresponding to the realizations of the interval Young's modulus which yield the a) LB and b) UB of the displacement in the $y$ - direction of node 20 , respectively $\left(C_{B}=0.05, l_{B}=L\right)$.

Figure 10. Interval stress component in the load direction of the plate under uniform traction evaluated at the integration points of a) FE 12 and b) FE 16: nominal value and bounds provided by the proposed method and the vertex method for $l_{B}=L$ and $C_{B}=0.05$.

Figure 11 a) Simply-supported plate with uncertain Young's modulus subjected to a downward uniformly distributed transverse load; b) FE mesh.

Figure 12. Proposed a) UB and b) LB of the normalized interval deflection of node A of the simply-supported plate versus the number $M$ of terms of the KL-like decomposition for different values of the parameter $l_{B}$.

Figure 13. Samples of the normalized interval Young's modulus $\left(C_{B}=0.05, l_{B}=0.5 L\right)$ which yield the LB of the normalized interval deflection at node A of the simply-supported plate.

Figure 14. Samples of the normalized interval Young's modulus $\left(C_{B}=0.05, l_{B}=0.5 L\right)$ which yield the UB of the normalized interval deflection at node A of the simply-supported plate.

Figure 15. Bounds of a) the normalized interval deflection of node A and b) the normalized interval rotation around the $x$-axis of node B of the simply-supported plate versus the ratio $l_{B} / L\left(C_{B}=0.05\right)$. 
Figure 16. Proposed coefficient of interval uncertainty of a) the normalized interval deflection of node $\mathrm{A}$ and $\mathrm{b}$ ) the normalized interval rotation around the $x$ - axis of node $\mathrm{B}$ of the simplysupported plate versus the ratio $l_{B} / L\left(M=10, C_{B}=0.05\right)$.

Figure 17. Samples of the normalized interval Young's modulus $\left(C_{B}=0.05, l_{B}=0.5 L\right)$ which yield the a) LB and b) UB of the normalized interval stress component $\tilde{\sigma}_{x x}^{I}$ in the $x$ - direction evaluated at the integration point 2 of FE 419 of the simply-supported plate.

Table captions 
Table 1. Bounds of the normalized interval deflection $\tilde{U}_{A z}^{I}$ of node A of the simply-supported plate provided by the proposed method and the vertex method, and associated absolute percentage errors $\left(l_{B}=0.5 L\right)$.

Table 2. Bounds of the normalized interval rotation around the $x$-axis of node B $\tilde{\varphi}_{B x}^{I}$ provided by the proposed method and the vertex method, and associated absolute percentage errors ( $\left.l_{B}=0.5 L\right)$

Table 3. Bounds of the normalized interval stress component $\tilde{\sigma}_{x x}^{I}$ evaluated at the integration points of FE 419 of the simply-supported plate provided by the proposed method and the vertex method, and associated absolute percentage errors $\left(C_{B}=0.05, l_{B}=0.5 L\right)$.

Table 4. Bounds of the normalized interval stress component $\tilde{\sigma}_{x x}^{I}$ in the $x$-direction evaluated at the integration points of FE 419 of the simply-supported plate provided by the proposed method and the vertex method, and associated absolute percentage errors $\left(C_{B}=0.1, l_{B}=0.5 L\right.$ ) .

\section{Nomenclature}


$\mathbf{A}_{i}$

$A_{i, j}$

$a_{0 i, j}$

$\mathbf{B}^{(h)}(\mathbf{x})$

$B^{I}(\mathbf{x})$

$B_{i, j}$

$\mathbf{b}(\mathbf{x}) \quad$ body forces

$C_{B} \quad$ parameter governing the degree of uncertainty of the interval field

c.i.u. [•] coefficient of interval uncertainty of •

$\mathbf{D}^{I}(\mathbf{x}) \quad$ interval constitutive matrix

$\mathbf{D}_{0} \quad$ nominal constitutive matrix

$\mathbf{D}_{i}(\mathbf{x}) \quad$ deviation matrix associated with the $i$-th extra unitary interval

$\mathbf{d}^{(h) I} \quad$ interval vector of the $h$-th element nodal displacements

$E_{0} \quad$ midpoint of $E^{I}(\mathbf{x})$

$E^{I}(\mathbf{x}) \quad$ interval Young's modulus field

$E^{(\mathrm{LB})}(x, y) \quad$ sample of Young's modulus which yields the lower bound of the response quantity of interest

$E^{(\mathrm{UB})}(x, y) \quad$ sample of Young's modulus which yields the upper bound of the response quantity of interest

$\hat{\mathbf{e}}^{I} \quad$ interval vector collecting $M$ extra unitary intervals

$\hat{e}_{i}^{I} \quad i-$ th extra unitary interval 
$\hat{\mathbf{e}}_{h, k}^{(\mathrm{LB})}$

$\hat{\mathbf{e}}_{h, k}^{(\mathrm{UB})}$

$F V^{l}$

$\mathbf{f}^{(h)}$

$\mathbf{K}^{I}$

$\mathbf{K}_{0}$

$\mathbf{K}_{i}$

$\mathbf{k}^{(h) I}$

$\mathbf{k}_{0}^{(h)}$

$\mathbf{k}_{i}^{(h)}$

vector of the combinations of the endpoints of the extra unitary intervals $\hat{e}_{h, k, i}^{(\mathrm{LB})}$

which give the lower bound of the $k$ - th component of the interval stress within the $h$-th finite element

vector of the combinations of the endpoints of the extra unitary intervals $\hat{e}_{h, k, i}^{(\mathrm{UB})}$

which give the upper bound of the $k$ - th component of the interval stress within the $h$-th finite element

global nodal force vector

interval field variable

nodal force vector of the $h$-th finite element

interval global stiffness matrix

global nominal stiffness matrix

$i-$ th global deviation stiffness matrix

$I$ (superscript) denotes interval variables

$\mathbf{L}^{(h)} \quad$ connectivity matrix

L $\quad$ plate width

$L_{e}^{I} \quad$ interval external work

$l_{B x}\left(l_{B}\right) \quad$ parameter ruling the spatial dependency of the uncertain property along the $x$ direction

$l_{B y}\left(l_{B}\right) \quad$ parameter ruling the spatial dependency of the uncertain property along the $y$ direction 


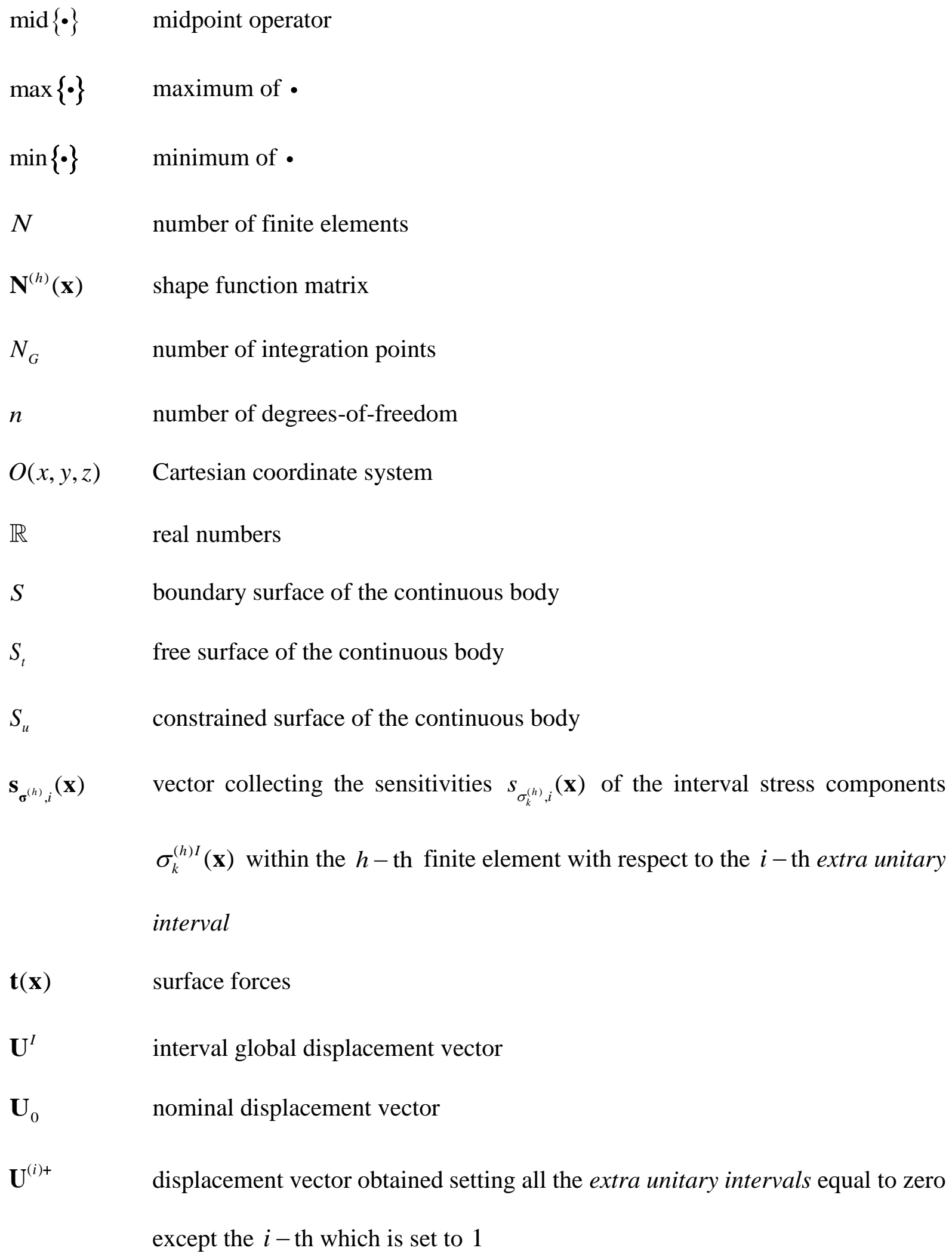




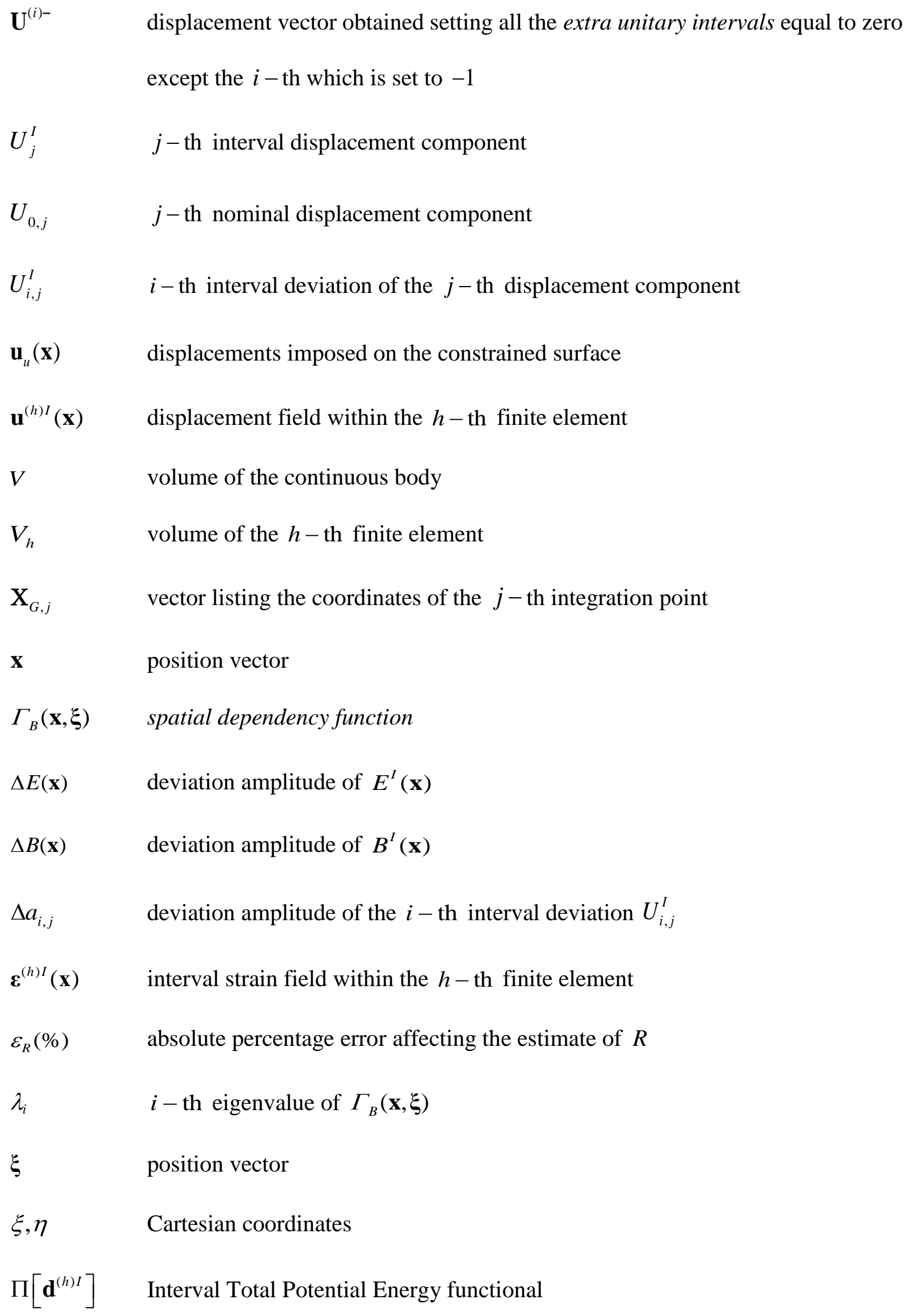


solution set

$\boldsymbol{\sigma}^{(h) I}(\mathbf{x}) \quad$ interval stress field within the $h$-th finite element

$\Phi^{I} \quad$ interval elastic strain energy

$\psi_{i}(\mathbf{x}) \quad i-$ th eigenfunction of $\Gamma_{B}(\mathbf{x}, \xi)$

$\cdot$ absolute value of $\bullet$

Overbar denotes the upper bound of an interval quantity

Underline denotes the lower bound of an interval quantity

Over tilde denotes normalized interval variables

\section{Acronyms and abbreviations}

c.i.u Coefficient of interval uncertainty

CIA Classical Interval Analysis

COSSAN Computational stochastic structural analysis

DOFs degrees-of-freedom

EUI Extra unitary interval

FEM Finite element method

FERUM Finite element reliability using MATLAB

FFEM Fuzzy finite element method

FV field variable

IDW Inverse Distance Weighting interpolation

IFEM Interval finite element method

IIA Improved interval analysis

IRSE Interval Rational Series Expansion 
ITPE Interval Total Potential Energy

KL Karhunen-Loève

LB Lower bound

LIFD Local Interval Field Decomposition method

NESSUS Numerical evaluation of stochastic structures under stress

SFEM Stochastic finite element method

StoFEL Stochastic finite element library

TSD Total spatial dependency

TSI Total spatial independency

UB Upper bound

UEL User Element subroutine

UMAT User MATerial

USDFLD USerDefinedFieLD 